
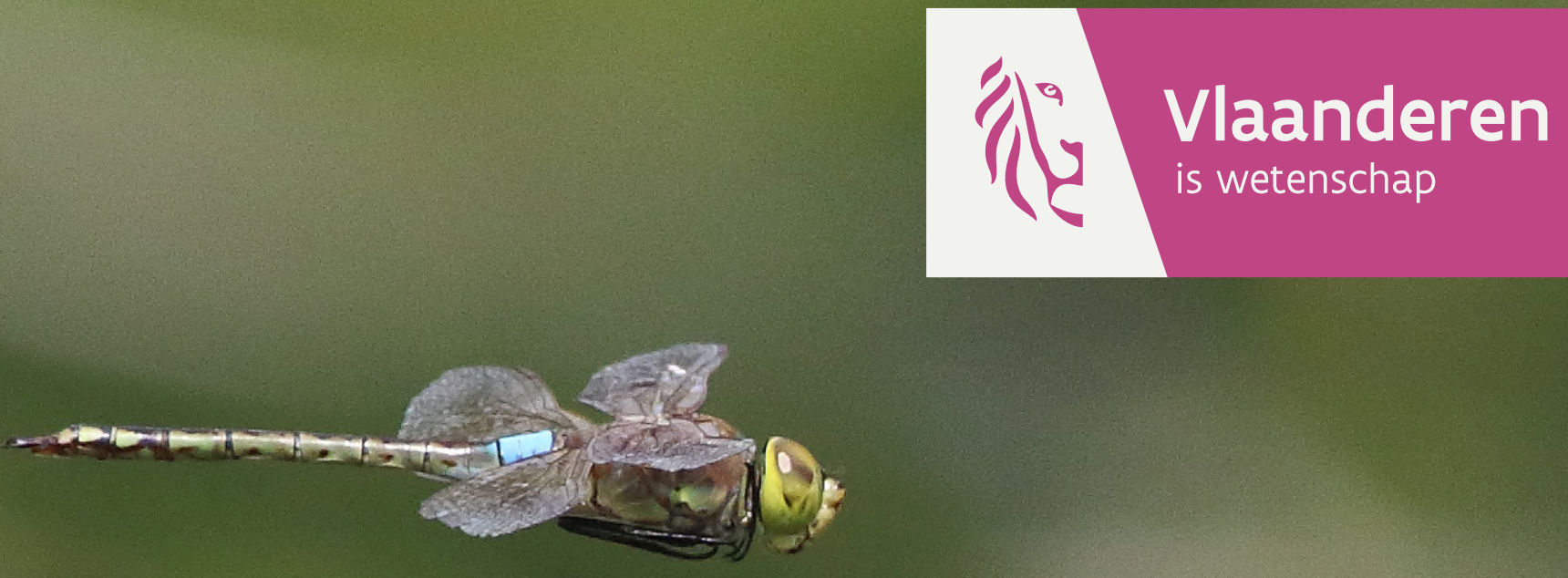

\title{
Trendbepaling natuurindicatoren 2019
}

Ivy Jansen

INSTITUUT

NATUUR- EN BOSONDERZOEK 
Auteurs:

Ivy Jansen

Instituut voor Natuur- en Bosonderzoek

Het INBO is het onafhankelijk onderzoeksinstituut van de Vlaamse overheid dat via toegepast wetenschappelijk onderzoek, data- en kennisontsluiting het biodiversiteits-beleid en -beheer onderbouwt en evalueert.

\section{Vestiging:}

Herman Teirlinckgebouw

INBO Brussel

Havenlaan 88 bus 73, 1000 Brussel

www.inbo.be

e-mail:

ivy.jansen@inbo.be

\section{Wijze van citeren:}

Jansen I.(2019). Trendbepaling natuurindicatoren 2019. Rapporten van het Instituut voor Natuur- en Bosonderzoek 2019 (32). Instituut voor Natuur- en Bosonderzoek, Brussel.

DOI: doi.org/10.21436/inbor.16703423

\section{D/2019/3241/197}

Rapporten van het Instituut voor Natuur- en Bosonderzoek 2019 (32)

ISSN: 1782-9054

Verantwoordelijke uitgever:

Maurice Hoffmann

\section{Foto cover:}

Zadellibel (Hemianax ephippiger)

(Geert De Knijf) 
INSTITUUT

NATUUR- EN

BOSONDERZOEK

\section{TRENDBEPALING NATUURINDICATOREN 2019}

Ivy Jansen

Rapporten van het Instituut voor Natuur- en Bosonderzoek 2019 (32)

Pure id 16703423 - doi.org/10.21436/inbor.16703423 - D/2019/3241/197 


\section{Dankwoord}

Met medewerking van: Geert De Knijf, Koen Devos, Beatrijs Van der Aa, Wouter Van Landuyt en Lieve Vriens 


\section{Samenvatting}

Dit rapport is een aanvulling op de website van de natuurindicatoren. Het bevat meer gedetailleerde informatie over de uitgevoerde trendanalyses, zoals het type model dat voor elke indicator gebruikt werd, samen met de output van dat statistische model, en een conclusie over het al dan niet significant zijn van de trend. 


\section{Inhoudsopgave}

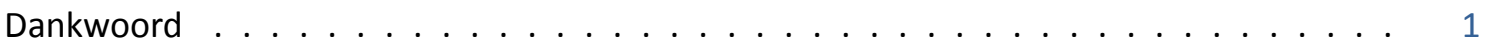

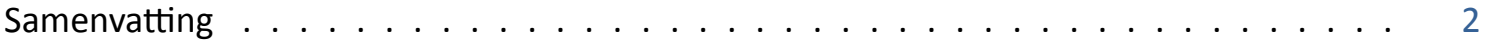

Inhoudsopgave ............................. . . . . . 4

1 Introductie ......................... 5

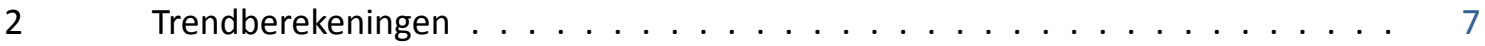

$2.1 \quad$ Overwinterende watervogelindex - totaal watervogels $\ldots \ldots \ldots \ldots \ldots$

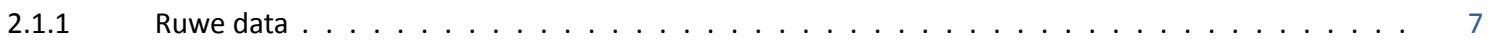

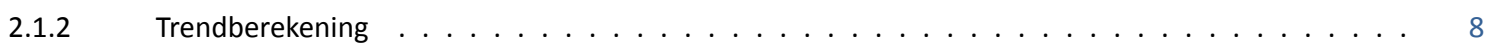

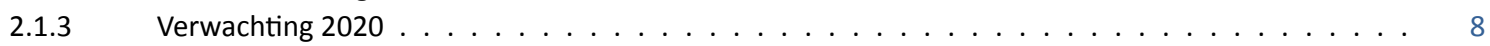

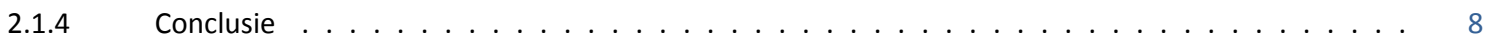

2.2 Trend Zuid-Europese libellensoorten - aantal soorten $\ldots \ldots \ldots \ldots$

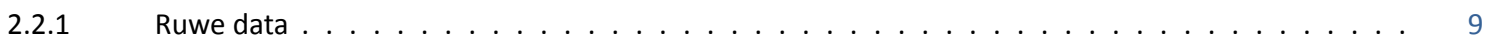

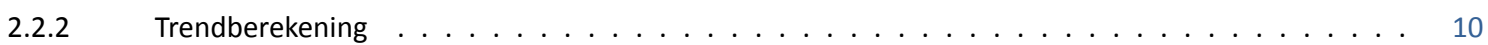

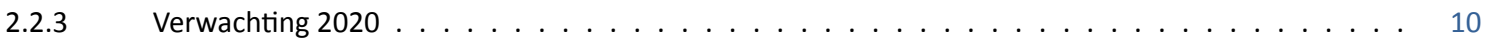

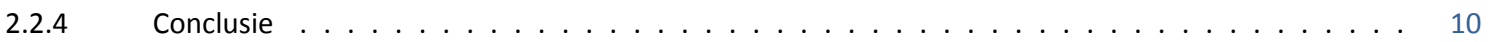

$2.3 \quad$ Trend Zuid-Europese libellensoorten - zwervende pantserjuffer ～. . . . . . . . . . . . . . 11

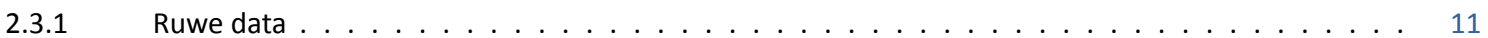

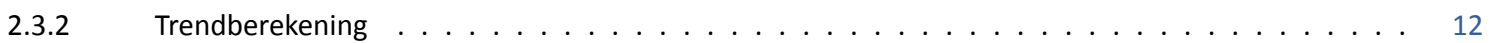

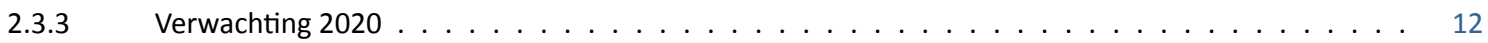

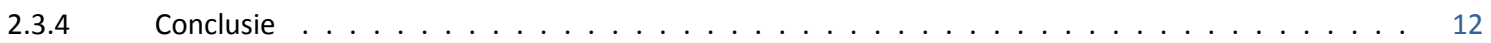

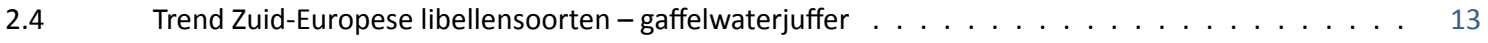

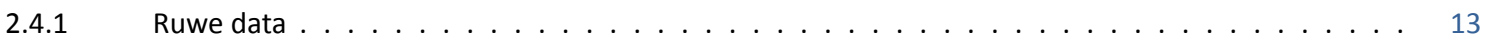

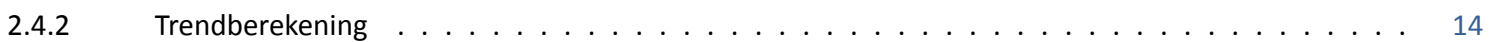

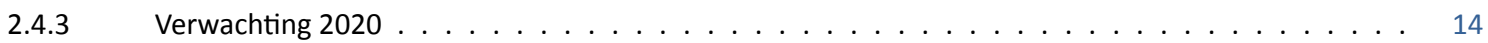

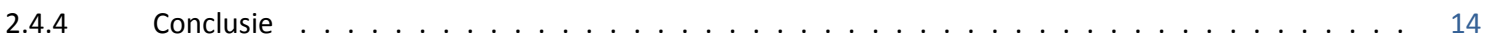

$2.5 \quad$ Trend Zuid-Europese libellensoorten -zuidelijke glazenmaker ～. . . . . . . . . . . . . . . 15

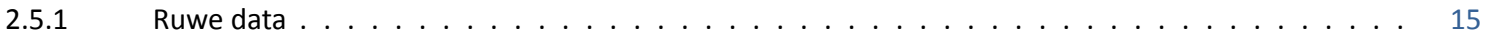

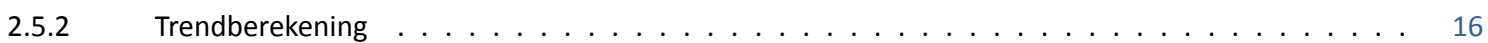

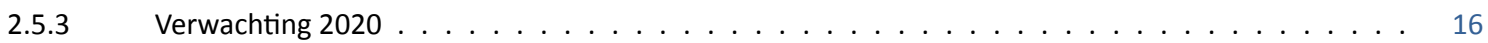

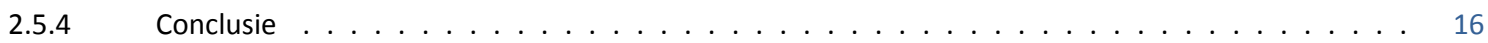

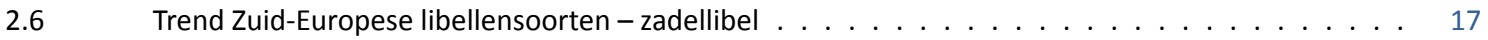

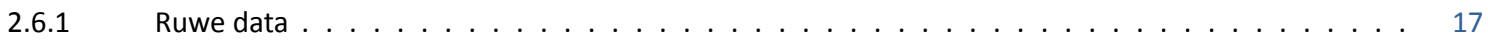

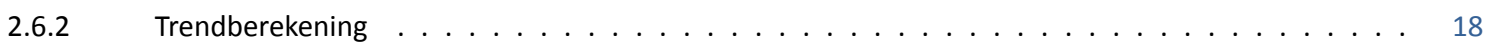

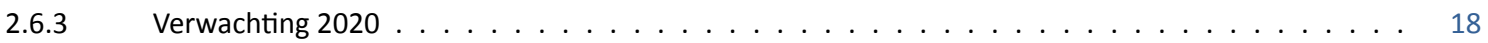

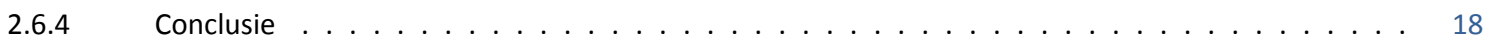

$2.7 \quad$ Trend Zuid-Europese libellensoorten -zuidelijke keizerlibel ～. . . . . . . . . . . . . . . . . 19

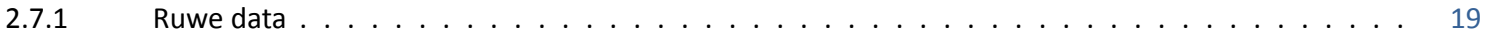

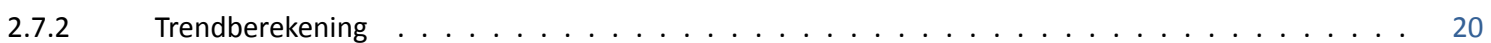

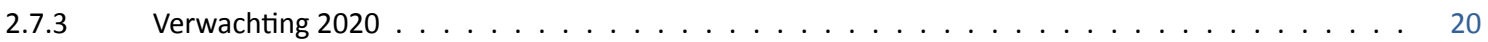

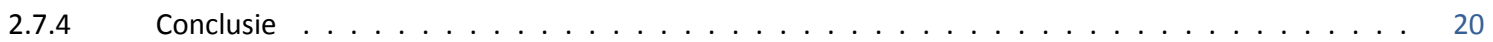

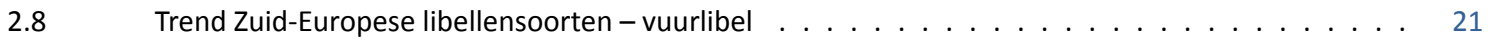

$2.8 .1 \quad$ Ruwe data . . . . . . . . . . . . . . . . . . . . . . . . . . . . . 21

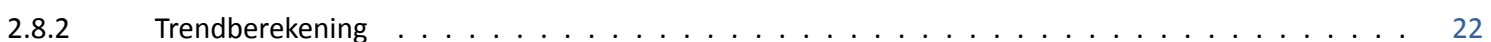

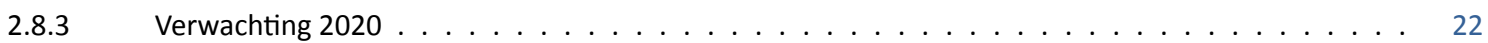

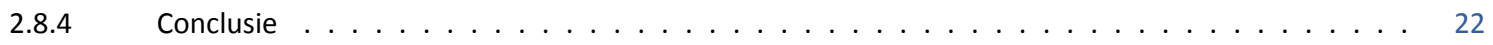

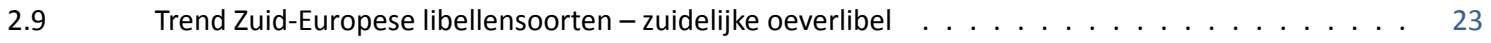

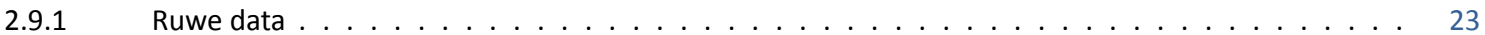




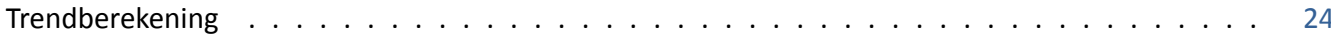

Verwachting 2020 ... . . . . . . . . . . . . . . . . . . . . . . . . . 24

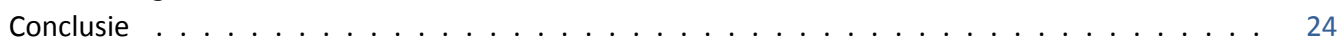

Trend Zuid-Europese libellensoorten -zuidelijke heidelibel $\ldots \ldots \ldots \ldots$

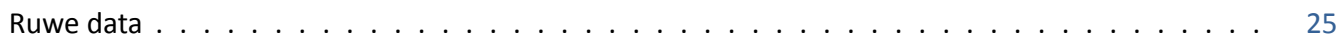

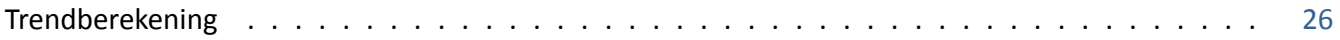

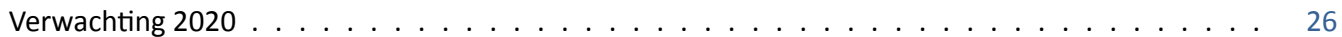

2.10 .3
$2.10 .4 \quad$ Conclusie

2.11 Trend Zuid-Europese libellensoorten - zwervende heidelibel . . . . . . . . . . . . . . . . . 27

2.11.1 Ruwe data . . . . . . . . . . . . . . . . . . . . . . . . . . 27

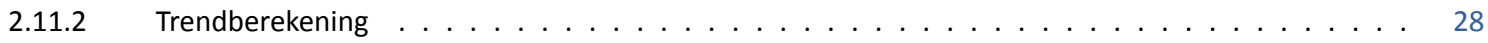

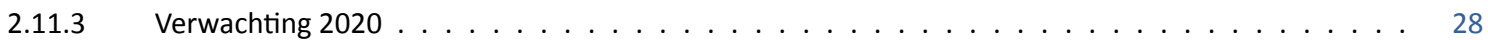

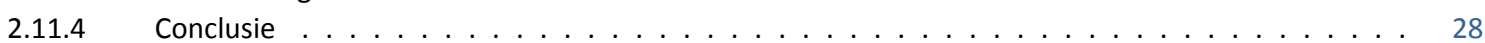

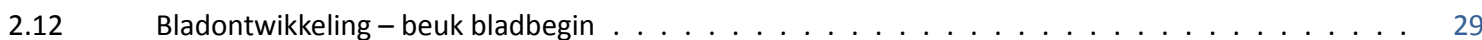

2.12.1 Ruwe data . . . . . . . . . . . . . . . . . . . . . . . . . . . 29

2.12.2 Trendberekening ............................... 29

2.12.3 Verwachting 2020 . . . . . . . . . . . . . . . . . . . . . . . . . . 30

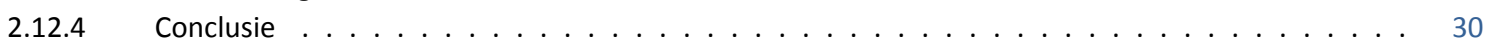

2.13 Bladontwikkeling - beuk bladvol . . . . . . . . . . . . . . . . . . . . . . . 31

2.13.1 Ruwe data . . . . . . . . . . . . . . . . . . . . . . . . . . . . 31

2.13.2 Trendberekening ............................... . . . . . . . . .

2.13.3 Verwachting 2020 ................................. 32

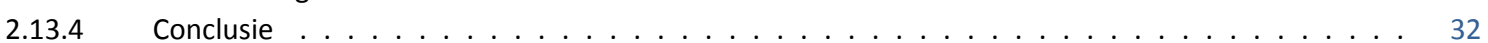

2.14 Bladontwikkeling - eik bladbegin . . . . . . . . . . . . . . . . . . . . . . 33

2.14.1 Ruwe data . . . . . . . . . . . . . . . . . . . . . . . . . . . . 33

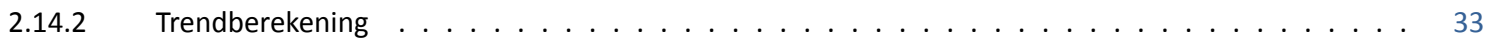

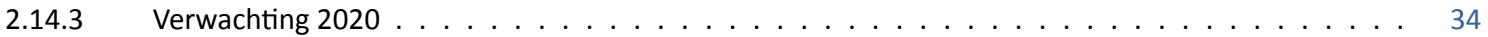

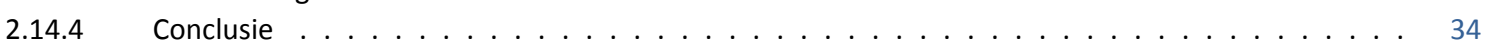

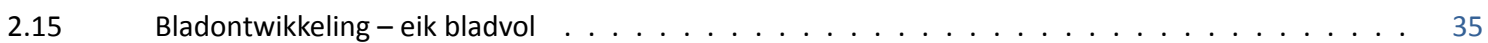

2.15.1 Ruwe data . . . . . . . . . . . . . . . . . . . . . . . . . 35

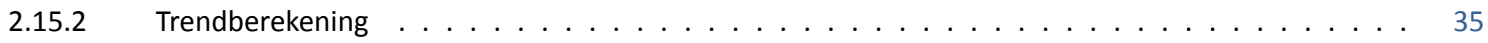

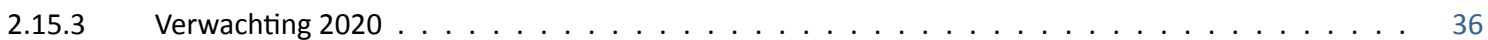

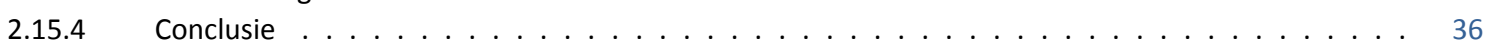

2.16 Piekmoment stuifmeelproductie bij berk en grassen - piek berk . . . . . . . . . . . . . . . . . . . 37

2.16.1 Ruwe data . . . . . . . . . . . . . . . . . . . . . . . . . . . . . 37

2.16.2 Trendberekening ............................... 38

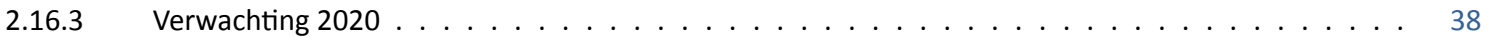

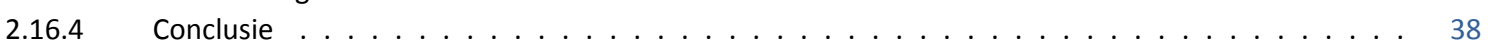

2.17 Piekmoment stuifmeelproductie bij berk en grassen - piek grassen . . . . . . . . . . . . . . . . . 39

2.17.1 Ruwe data . . . . . . . . . . . . . . . . . . . . . . . . . . . . 39

2.17.2 Trendberekening ................................ 40

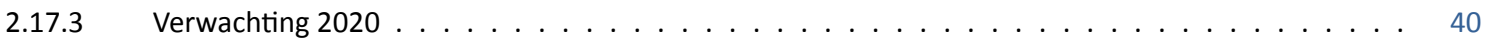

2.17.4 Conclusie ..................................440

2.18 Bedreiging door uitheemse plantensoorten - aandeel uitheemse planten . . . . . . . . . . . 41

2.18.1 Ruwe data . . . . . . . . . . . . . . . . . . . . . . . . . . . . . 41

2.18.2 Trendberekening .................................42

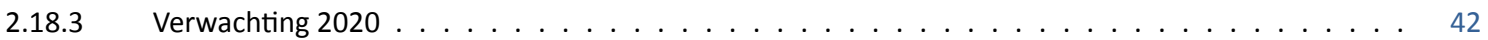

2.18.4 Conclusie ................................43 


\section{INTRODUCTIE}

De NARA natuurindicatoren kunnen in 3 grote groepen onderverdeeld worden op basis van de gemeten eenheid:

1. aantallen (Poisson verdeelde gegevens)

2. percentages (binomiaal verdeelde gegevens)

3. oppervlakte, lengte, index (normaal verdeelde gegevens)

Voor elk type indicator is een andere aanpak van de trendberekening noodzakelijk:

1. $Y$ = aantal

Poisson regressie

$$
\log (Y)=a+b X
$$

2. $Y=1$ (succes) of $Y=0$ (faling), met $\pi=P(Y=1)=$ kans op succes

Logistische regressie

$$
\operatorname{logit}(\pi)=\log \left(\frac{\pi}{1-\pi}\right)=a+b X
$$

3. $Y=$ continue variabele (normaal verdeeld)

lineaire regressie

$$
Y=a+b X+\epsilon
$$

of kwadratische regressie

$$
Y=a+b X+c X^{2}+\epsilon
$$

of kubische regressie

$$
Y=a+b X+c X^{2}+d X^{3}+\epsilon
$$

of exponentiële regressie

$$
\log (Y+1)=a+b X+\epsilon
$$

waarbij telkens $X=$ Jaartal. In het derde geval wordt op basis van statistische testen het beste model geselecteerd.

Voor grote aantallen (zoals Ledenaantal) is het niet noodzakelijk om een Poisson regressie uit te voeren, maar kan in vele gevallen een normale benadering gebruikt worden, en kunnen we overschakelen naar de derde methode.

Omdat voor bepaalde indicatoren het gekozen model een te sterke vereenvoudiging lijkt van de werkelijke trend, bekijken we ook het model met een smoother doorheen de tijd.

$$
f(Y)=a+s(\text { Jaartal, } k)+\epsilon
$$


waarbij het aantal knopen $k$ wel beperkt wordt tot het minimum van 4 en het aantal observaties gedeeld door 2, om te vermijden dat er oversmoothing optreedt. Een optimalisatiemethode bepaalt uiteindelijk het aantal vrijheidsgraden (edf) voor deze smoother. Ook voor de smoother kiezen we de gepaste verdeling voor de respons via de link-functie $f$ (log-link voor Poisson, logit-link voor binomiaal, identity-link voor de normale verdeling). Deze smoother geeft voor continue variabelen ook een indicatie welke macht van Jaartal opgenomen moet worden in het model.

Voor elke indicator worden de schattingen van de modelparameters weergegeven, samen met hun significantie. Voor de "gewone" modellen werd voor de eenvoud van interpretatie van de parameterschattingen jaar steeds herschaald zodat de start van de metingen als referentie genomen wordt, dwz. dat voor de start van de studie jaar $=0$, het volgende jaar jaar $=1, \ldots$. Dit geeft als voordeel dat het intercept $(a)$ van het model altijd de startwaarde van de indicator is, en de helling van de rechte $(b)$ de jaarlijkse wijziging t.o.v. de start weergeeft. Voor de modellen met smoother gebeurde deze herschaling niet, aangezien daar het intercept steeds de gemiddelde respons is. Voor de smoothers s(Jaartal) zijn de waarden onder Estimate en Std..Error respectievelijk edf (estimated degrees of freedom) en res.df (estimated residual degrees of freedom).

Tevens worden per indicator 2 figuren getoond:

- scatterplot van de gegevens

- berekende trend met een $95 \%$ betrouwbaarheidsinterval voor de trendlijn

Uiteindelijk wordt er ook nog een verwachting voor 2020 gegeven. Dit is niets anders dan een predictie interval voor het jaar 2020, bekomen uit het gekozen model. 


\section{TRENDBEREKENINGEN}

\subsection{OVERWWINTTERENDE WATERVOGGELINDPEX-TOTTAAL WATERVOGGELSS}

\subsubsection{Ruwe data}

\begin{tabular}{rr}
\hline Jaartal & index \\
\hline 1991 & 100.00 \\
1992 & 156.70 \\
1993 & 177.00 \\
1994 & 244.60 \\
1995 & 297.70 \\
1996 & 259.00 \\
1997 & 229.90 \\
1998 & 322.30 \\
1999 & 316.30 \\
2000 & 397.00 \\
2001 & 478.80 \\
2002 & 531.00 \\
2003 & 425.00 \\
2004 & 452.00 \\
2005 & 485.00 \\
2006 & 396.00 \\
2007 & 403.00 \\
2008 & 376.00 \\
2009 & 422.00 \\
2010 & 384.00 \\
2011 & 349.00 \\
2013 & 408.00 \\
2014 & 359.00 \\
2015 & 385.00 \\
2016 & 387.00 \\
2017 & 367.00 \\
2018 & 390.00 \\
\hline \hline &
\end{tabular}


Overwinterende watervogelindex totaal watervogels

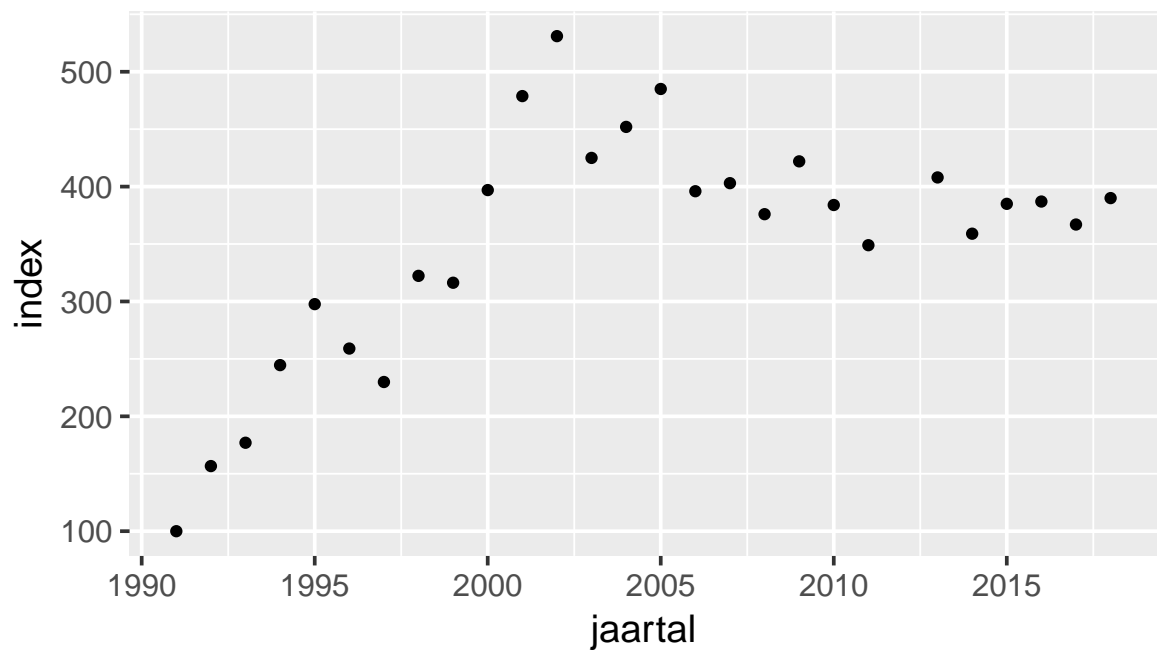

\subsubsection{Trendberekening}

Methode: Derdemacht

Parameterschattingen

params Estimate Std..Error statistic p.value 1 Intercept $79.3101158531 .22557196 \quad 2.539909 \quad 1.830443 \mathrm{e}-02$

2 cJaar $59.88311651 \quad 10.30908845 \quad 5.808769 \quad 6.427319 \mathrm{e}-06$

cJaar2 $-3.27156662 \quad 0.90409495 \quad-3.618610 \quad 1.442723 e-03$

$\begin{array}{llllll}\text { cJaar3 } & 0.05394169 & 0.02203151 & 2.448388 & 2.239456 \mathrm{e}-02\end{array}$

Overwinterende watervogelindex totaal watervogels

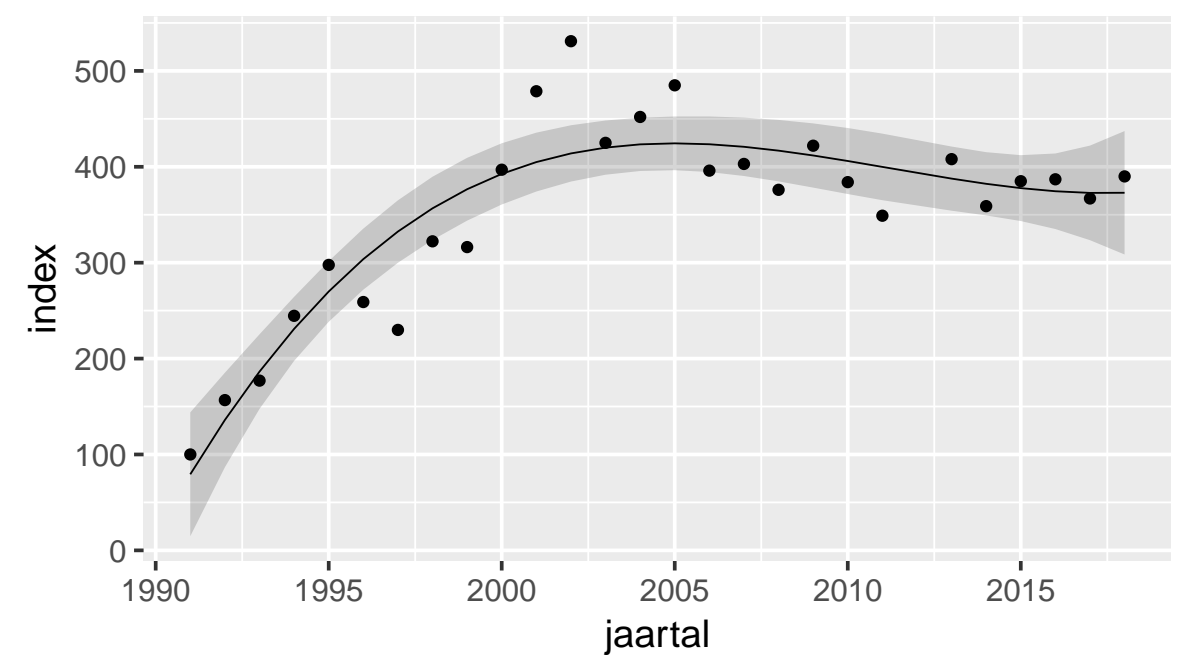

\subsubsection{Verwachting 2020}

$[234,526]$

\subsubsection{Conclusie}

Significante trend. Stijging tot 2005, sindsdien lichte afname. 


\subsection{TREND ZUID-EUROPESE LIBELLENSSOORTEN - AANTAL SOORTEN}

\subsubsection{Ruwe data}

\begin{tabular}{rr}
\hline Jaartal & aantal \\
\hline 1980 & 0.00 \\
1981 & 0.00 \\
1982 & 0.00 \\
1983 & 0.00 \\
1984 & 3.00 \\
1985 & 1.00 \\
1987 & 1.00 \\
1989 & 2.00 \\
1990 & 1.00 \\
1991 & 3.00 \\
1992 & 2.00 \\
1993 & 2.00 \\
1994 & 5.00 \\
1995 & 5.00 \\
1996 & 5.00 \\
1997 & 5.00 \\
1998 & 5.00 \\
1999 & 5.00 \\
2000 & 6.00 \\
2001 & 5.00 \\
2002 & 6.00 \\
2003 & 8.00 \\
2004 & 8.00 \\
2005 & 7.00 \\
2006 & 8.00 \\
2007 & 8.00 \\
2008 & 9.00 \\
2009 & 8.00 \\
2010 & 8.00 \\
2011 & 8.00 \\
2012 & 8.00 \\
2013 & 8.00 \\
2014 & 8.00 \\
2015 & 9.00 \\
2016 & 10.00 \\
\hline & 9.00 \\
2017 & \\
200 \\
\hline
\end{tabular}




\section{Trend Zuid-Europese libellensoorten}

aantal soorten

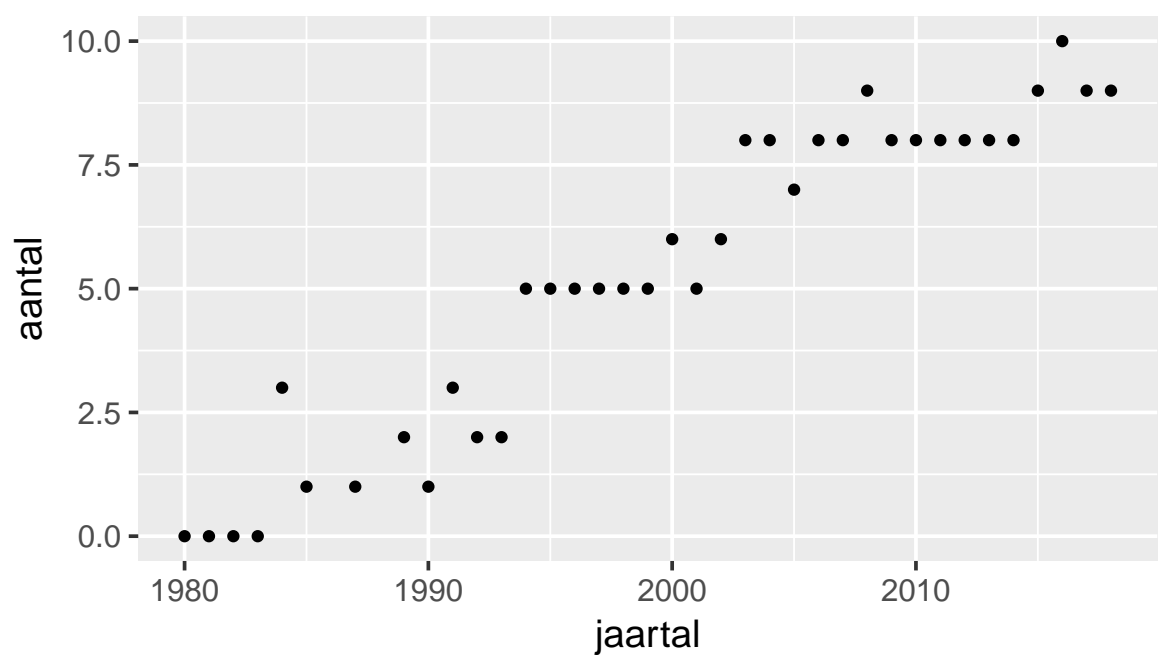

\subsubsection{Trendberekening}

Methode: Poisson smoother

Parameterschattingen

params Estimate Std..Error statistic p.value

1 Intercept $1.364298 \quad 0.102146 \quad 13.35634 \quad 1.087761 \mathrm{e}-40$

$2 \mathrm{~s}$ (Jaartal) $2.361727 \quad 2.721344 \quad 50.92207 \quad 1.133454 \mathrm{e}-10$

Trend Zuid-Europese libellensoorten aantal soorten

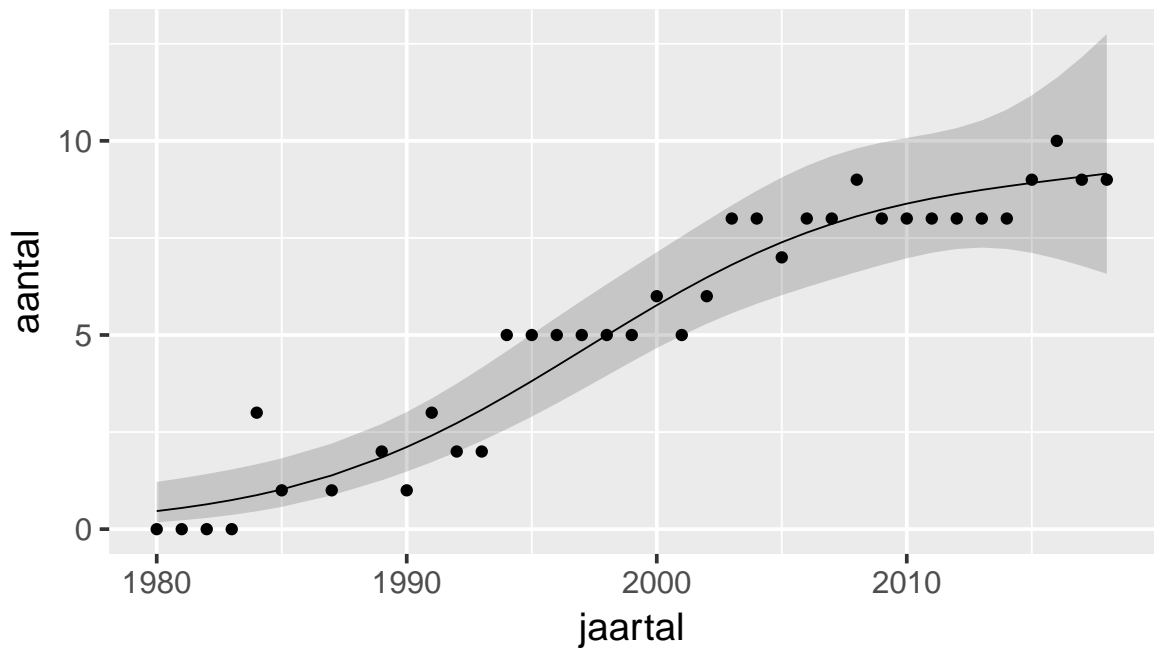

\subsubsection{Verwachting 2020}

$[6.15,14.1]$

\subsubsection{Conclusie}

Significante toename. 


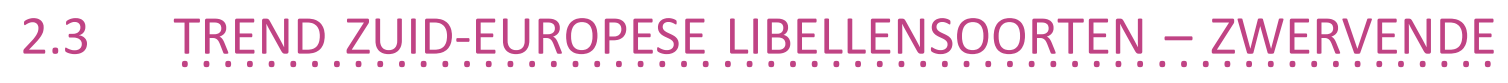
PANTSERJUFFER

\subsubsection{Ruwe data}

\begin{tabular}{rr}
\hline Jaartal & aantal \\
\hline 1980 & 0.00 \\
1981 & 0.00 \\
1982 & 0.00 \\
1983 & 0.00 \\
1984 & 1.00 \\
1985 & 3.00 \\
1987 & 0.00 \\
1989 & 0.00 \\
1990 & 0.00 \\
1991 & 0.00 \\
1992 & 0.00 \\
1993 & 0.00 \\
1994 & 5.00 \\
1995 & 55.00 \\
1996 & 12.00 \\
1997 & 30.00 \\
1998 & 17.00 \\
1999 & 26.00 \\
2000 & 24.00 \\
2001 & 7.00 \\
2002 & 13.00 \\
2003 & 23.00 \\
2004 & 17.00 \\
2005 & 22.00 \\
2006 & 45.00 \\
2007 & 33.00 \\
2008 & 35.00 \\
2009 & 30.00 \\
2010 & 58.00 \\
2011 & 36.00 \\
2012 & 48.00 \\
2013 & 64.00 \\
2014 & 53.00 \\
2015 & 31.00 \\
2016 & 84.00 \\
2017 & 59.00 \\
2018 & 123.00 \\
\hline \hline & \\
\hline
\end{tabular}




\section{Trend Zuid-Europese libellensoorten}

zwervende pantserjuffer

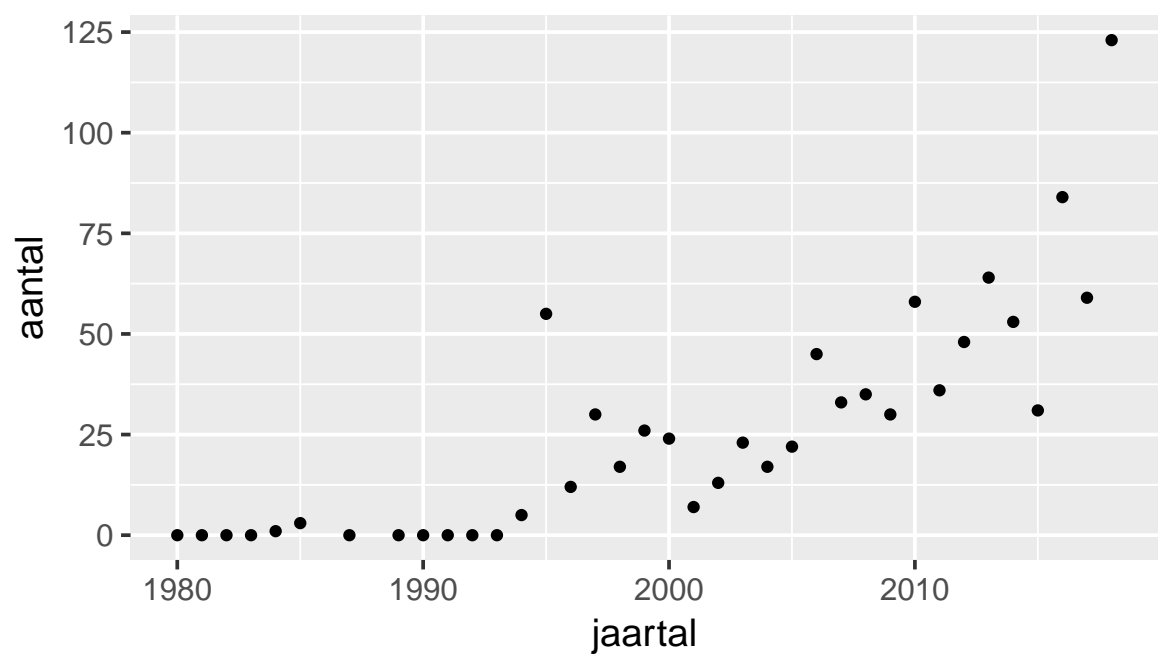

\subsubsection{Trendberekening}

Methode: Poisson smoother

Parameterschattingen

params Estimate Std..Error statistic p.value

$1 \quad$ Intercept $2.090912 \quad 0.1452777 \quad 14.39252 \quad 5.765913 \mathrm{e}-47$

2 s(Jaartal) $2.971743 \quad 2.9992850 \quad 427.38967 \quad 7.250568 \mathrm{e}-91$

Trend Zuid-Europese libellensoorten

zwervende pantserjuffer

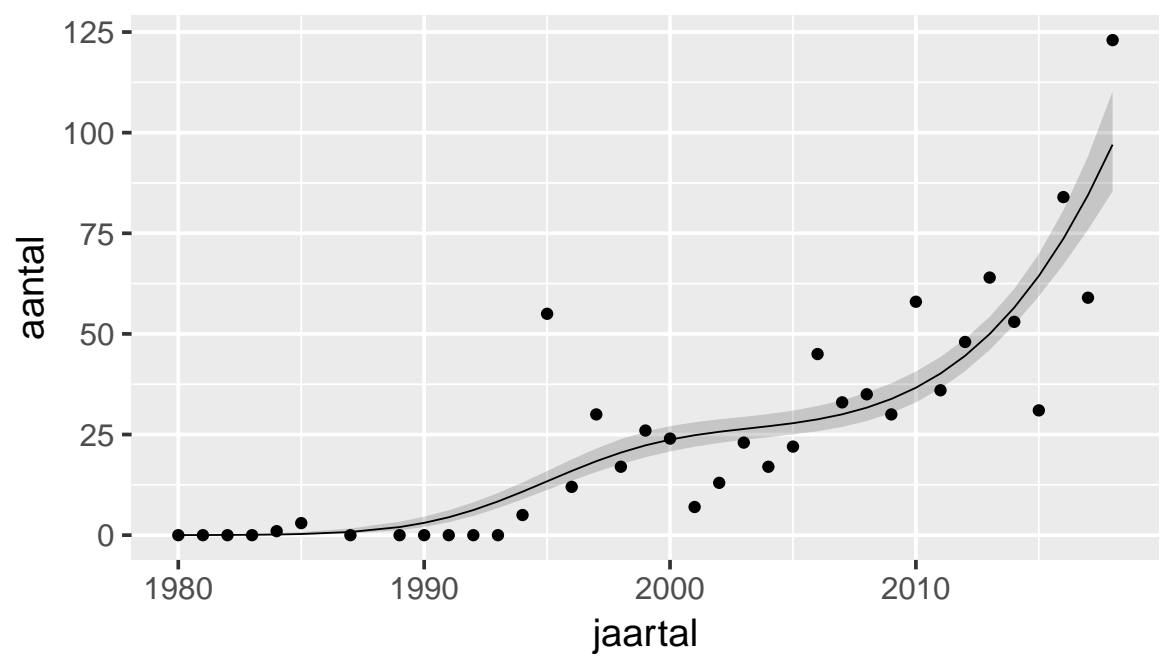

\subsubsection{Verwachting 2020}

$[108,152]$

\subsubsection{Conclusie}

Significante exponentiële toename. 


\subsection{TREND ZUID-EUROPESE LIBELLENSSOORTEN - GAFFELWATERJUYFFER}

\subsubsection{Ruwe data}

\begin{tabular}{rr}
\hline Jaartal & aantal \\
\hline 1980 & 0.00 \\
1981 & 0.00 \\
1982 & 0.00 \\
1983 & 0.00 \\
1984 & 0.00 \\
1985 & 0.00 \\
1987 & 0.00 \\
1989 & 0.00 \\
1990 & 0.00 \\
1991 & 0.00 \\
1992 & 0.00 \\
1993 & 0.00 \\
1994 & 0.00 \\
1995 & 0.00 \\
1996 & 0.00 \\
1997 & 0.00 \\
1998 & 0.00 \\
1999 & 1.00 \\
2000 & 0.00 \\
2001 & 0.00 \\
2002 & 0.00 \\
2003 & 1.00 \\
2004 & 1.00 \\
2005 & 0.00 \\
2006 & 4.00 \\
2007 & 15.00 \\
2008 & 23.00 \\
2009 & 37.00 \\
2010 & 52.00 \\
2011 & 49.00 \\
2012 & 57.00 \\
2013 & 69.00 \\
2014 & 80.00 \\
2015 & 88.00 \\
2016 & 128.00 \\
2017 & 138.00 \\
2018 & 221.00 \\
\hline \hline & \\
\hline
\end{tabular}


Trend Zuid-Europese libellensoorten gaffelwaterjuffer

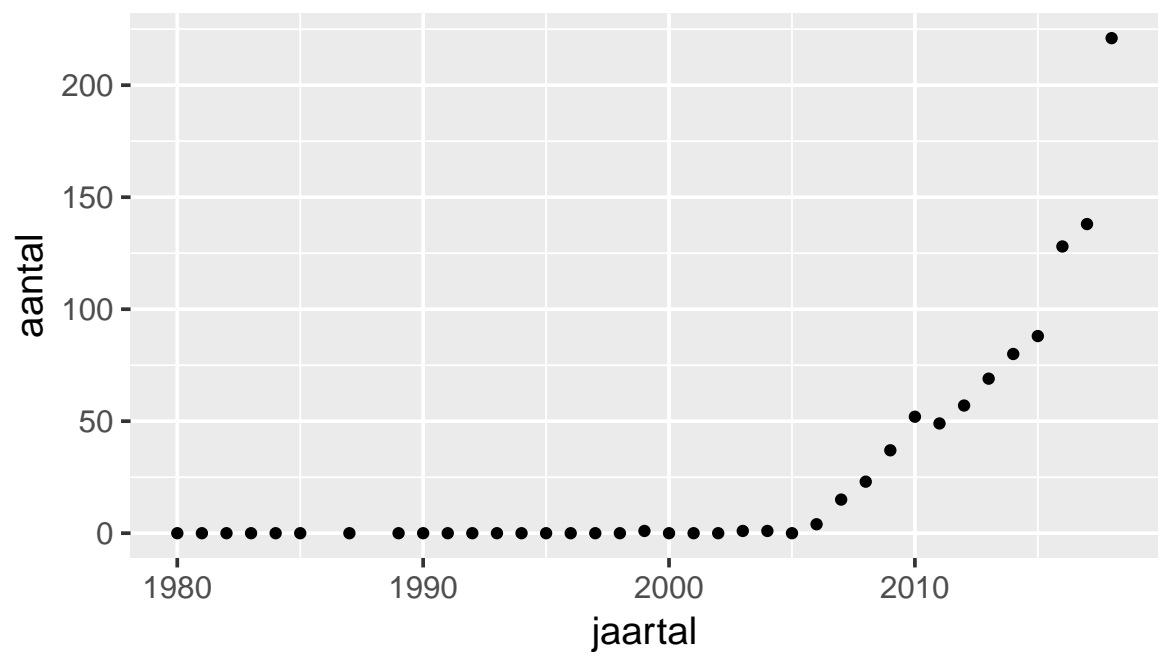

\subsubsection{Trendberekening}

Methode: Poisson smoother

Parameterschattingen

params Estimate Std..Error statistic p.value

1 Intercept $-20.312255 \quad 10.035660 \quad-2.024008 \quad 4.296935 \mathrm{e}-02$

$2 \mathrm{~s}$ (Jaartal) $2.785738 \quad 2.954249566 .484816 \quad 9.260846 \mathrm{e}-101$

Trend Zuid-Europese libellensoorten

gaffelwaterjuffer

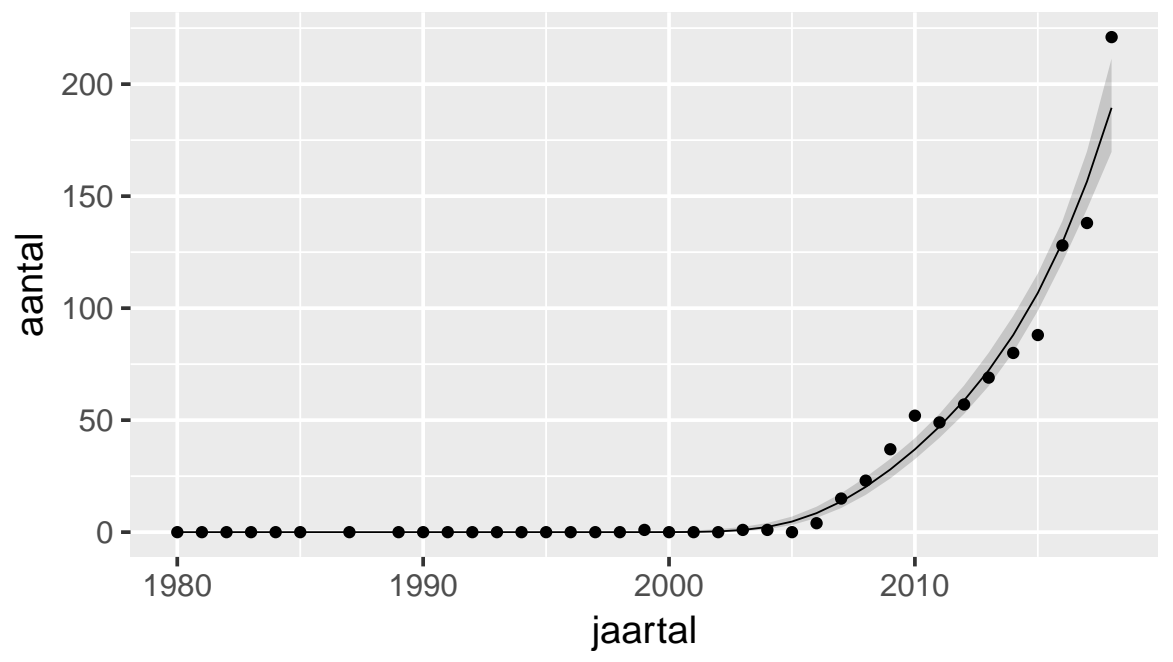

\subsubsection{Verwachting 2020}

$[231,333]$

\subsubsection{Conclusie}

Significante exponentiële toename. 


\subsection{TRENDZZUID-EUROPESE LIBELLENSOORTEN -ZUIDELLIKE GLAZENMAKER}

\subsubsection{Ruwe data}

\begin{tabular}{|c|c|}
\hline Jaartal & aantal \\
\hline 1980 & 0.00 \\
\hline 1981 & 0.00 \\
\hline 1982 & 0.00 \\
\hline 1983 & 0.00 \\
\hline 1984 & 0.00 \\
\hline 1985 & 0.00 \\
\hline 1987 & 0.00 \\
\hline 1989 & 0.00 \\
\hline 1990 & 0.00 \\
\hline 1991 & 1.00 \\
\hline 1992 & 0.00 \\
\hline 1993 & 0.00 \\
\hline 1994 & 1.00 \\
\hline 1995 & 17.00 \\
\hline 1996 & 2.00 \\
\hline 1997 & 2.00 \\
\hline 1998 & 1.00 \\
\hline 1999 & 0.00 \\
\hline 2000 & 2.00 \\
\hline 2001 & 1.00 \\
\hline 2002 & 1.00 \\
\hline 2003 & 7.00 \\
\hline 2004 & 8.00 \\
\hline 2005 & 8.00 \\
\hline 2006 & 13.00 \\
\hline 2007 & 0.00 \\
\hline 2008 & 7.00 \\
\hline 2009 & 14.00 \\
\hline 2010 & 18.00 \\
\hline 2011 & 0.00 \\
\hline 2012 & 3.00 \\
\hline 2013 & 15.00 \\
\hline 2014 & 12.00 \\
\hline 2015 & 8.00 \\
\hline 2016 & 12.00 \\
\hline 2017 & 13.00 \\
\hline 2018 & 54.00 \\
\hline
\end{tabular}


Trend Zuid-Europese libellensoorten zuidelijke glazenmaker

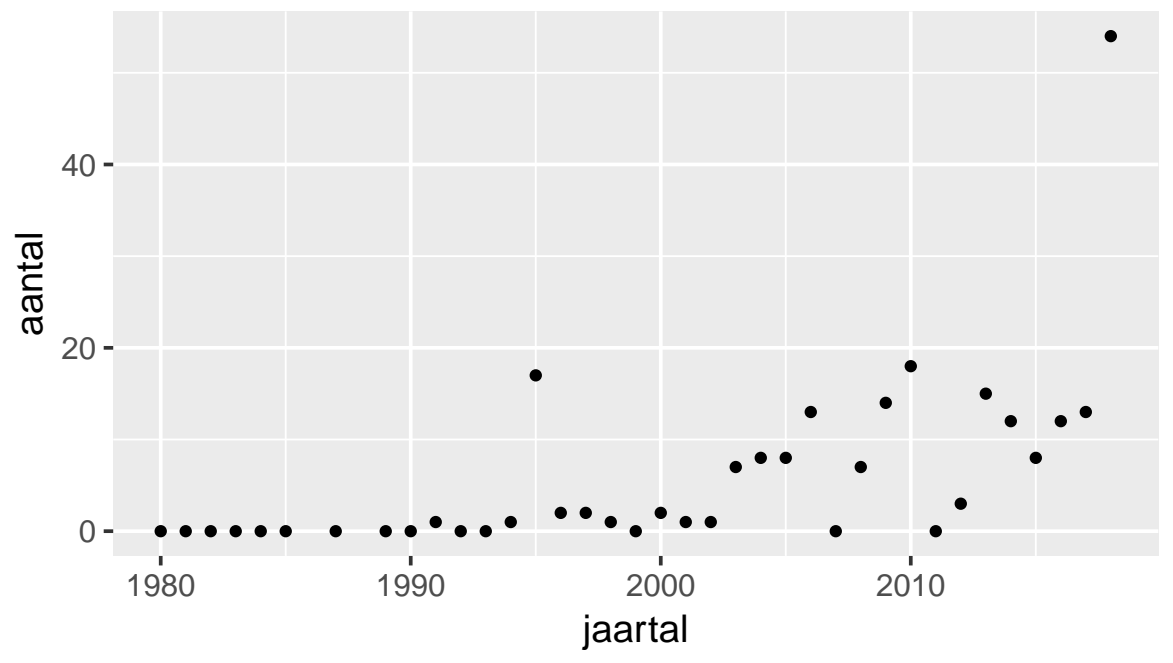

\subsubsection{Trendberekening}

Methode: Poisson smoother

Parameterschattingen

params Estimate Std..Error statistic p.value

1 Intercept $0.4058825 \quad 0.3422024 \quad 1.186089 \quad 2.355871 \mathrm{e}-01$

2 s(Jaartal) $2.90687892 .9921766 \quad 154.8338719 .714859 \mathrm{e}-32$

Trend Zuid-Europese libellensoorten zuidelijke glazenmaker

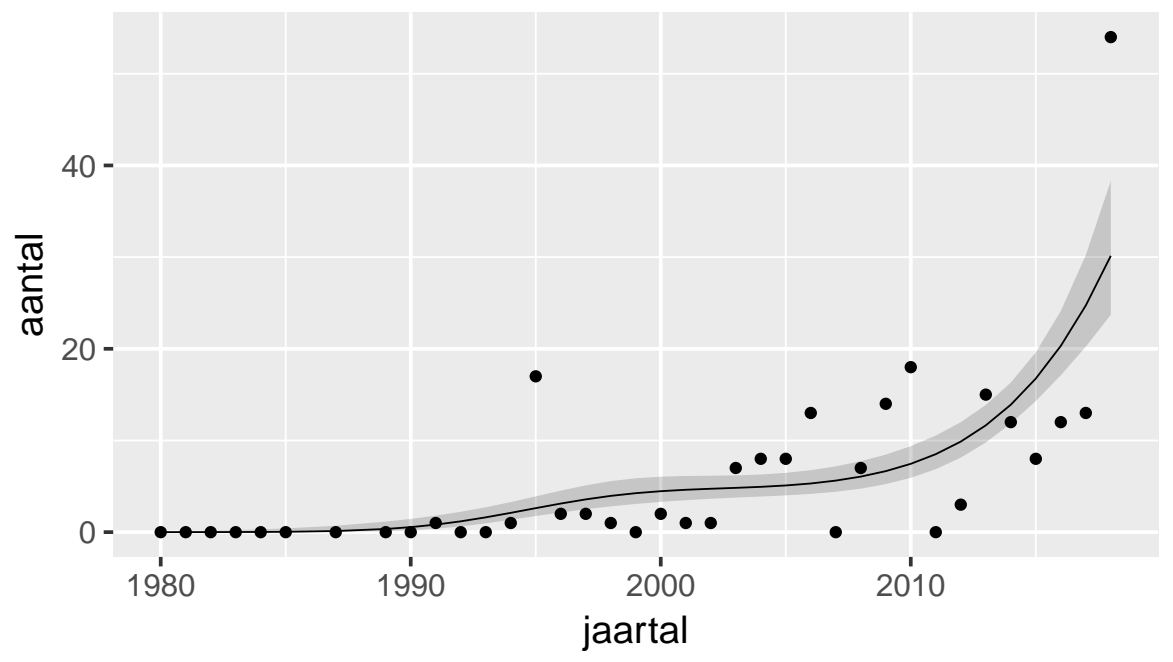

\subsubsection{Verwachting 2020}

$[32.1,62.5]$

\subsubsection{Conclusie}

Significante exponentiële toename. 


\subsection{TREND ZUID-EUROPESE LIBELLENSOORTEN - ZADELLIBEL}

\subsubsection{Ruwe data}

\begin{tabular}{rr}
\hline Jaartal & aantal \\
\hline 1980 & 0.00 \\
1981 & 0.00 \\
1982 & 0.00 \\
1983 & 0.00 \\
1984 & 0.00 \\
1985 & 0.00 \\
1987 & 0.00 \\
1989 & 0.00 \\
1990 & 0.00 \\
1991 & 0.00 \\
1992 & 0.00 \\
1993 & 0.00 \\
1994 & 0.00 \\
1995 & 0.00 \\
1996 & 0.00 \\
1997 & 0.00 \\
1998 & 1.00 \\
1999 & 0.00 \\
2000 & 0.00 \\
2001 & 0.00 \\
2002 & 0.00 \\
2003 & 0.00 \\
2004 & 0.00 \\
2005 & 1.00 \\
2006 & 0.00 \\
2007 & 1.00 \\
2008 & 1.00 \\
2009 & 0.00 \\
2010 & 0.00 \\
2011 & 7.00 \\
2012 & 0.00 \\
2013 & 0.00 \\
2014 & 0.00 \\
2015 & 7.00 \\
\hline & 1.00 \\
2016 & 3.00 \\
\hline
\end{tabular}


Trend Zuid-Europese libellensoorten zadellibel

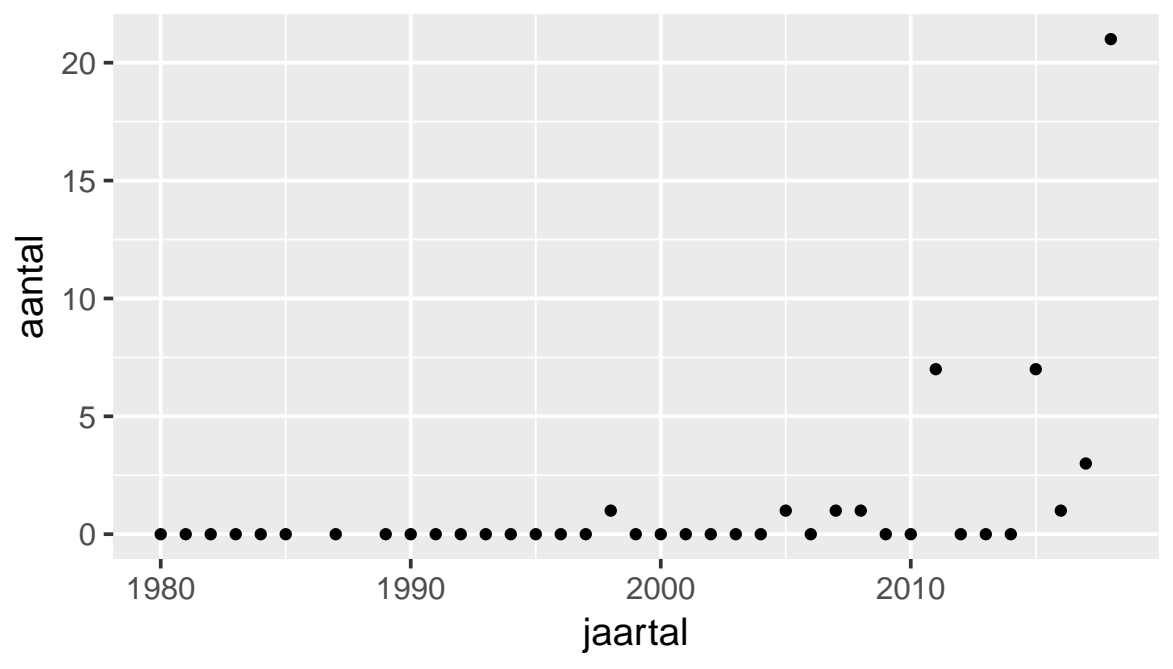

\subsubsection{Trendberekening}

Methode: Poisson smoother

Parameterschattingen

params Estimate Std..Error statistic p.value

1 Intercept $-2.453398 \quad 0.904871 \quad-2.711324 \quad 6.701516 \mathrm{e}-03$

$2 \mathrm{~s}$ (Jaartal) $1.334311 \quad 1.571120 \quad 19.442628 \quad 2.784294 \mathrm{e}-05$

Trend Zuid-Europese libellensoorten zadellibel

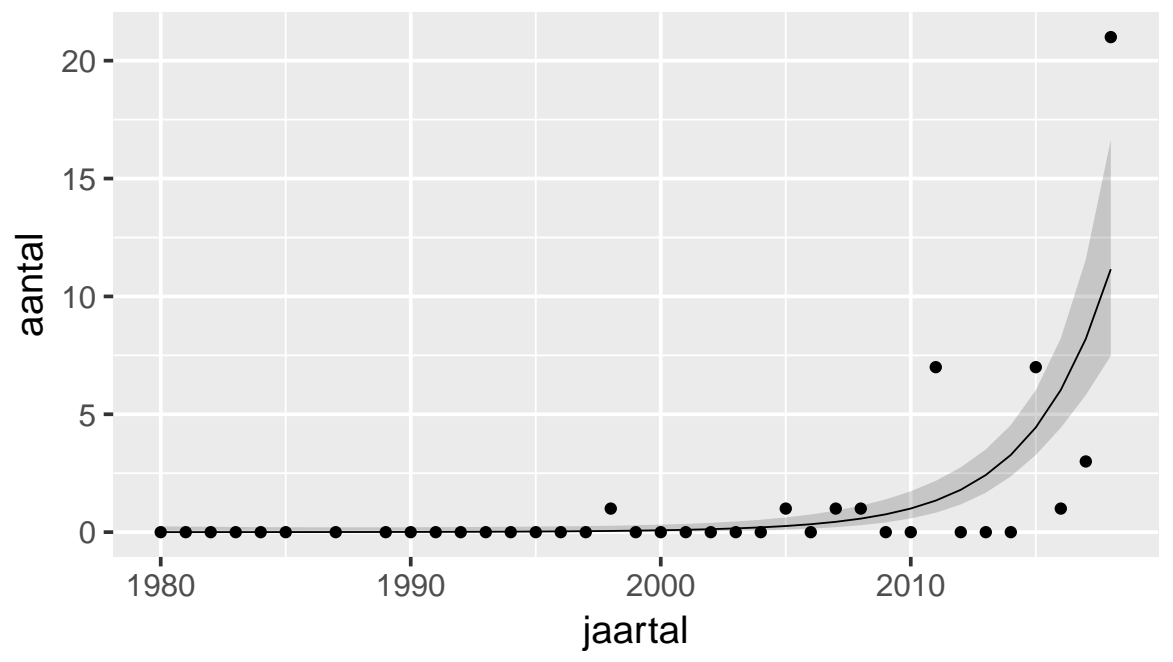

\subsubsection{Verwachting 2020}

$[11.9,35.7]$

\subsubsection{Conclusie}

Significante exponentiële toename. 


\subsection{TREND ZUID-EUROPESE LIBELLENSOORTEN -ZUIDELIJKE KEIZERLIBEL}

\subsubsection{Ruwe data}

\begin{tabular}{rr}
\hline Jaartal & aantal \\
\hline 1980 & 0.00 \\
1981 & 0.00 \\
1982 & 0.00 \\
1983 & 0.00 \\
1984 & 1.00 \\
1985 & 0.00 \\
1987 & 0.00 \\
1989 & 0.00 \\
1990 & 0.00 \\
1991 & 0.00 \\
1992 & 0.00 \\
1993 & 0.00 \\
1994 & 0.00 \\
1995 & 0.00 \\
1996 & 0.00 \\
1997 & 0.00 \\
1998 & 0.00 \\
1999 & 4.00 \\
2000 & 3.00 \\
2001 & 0.00 \\
2002 & 1.00 \\
2003 & 5.00 \\
2004 & 2.00 \\
2005 & 12.00 \\
2006 & 9.00 \\
2007 & 5.00 \\
2008 & 15.00 \\
2009 & 10.00 \\
2010 & 23.00 \\
2011 & 24.00 \\
2012 & 23.00 \\
2013 & 40.00 \\
2014 & 48.00 \\
2015 & 73.00 \\
2016 & 71.00 \\
2017 & 105.00 \\
2018 & 161.00 \\
\hline \hline & \\
\hline
\end{tabular}


Trend Zuid-Europese libellensoorten zuidelijke keizerlibel

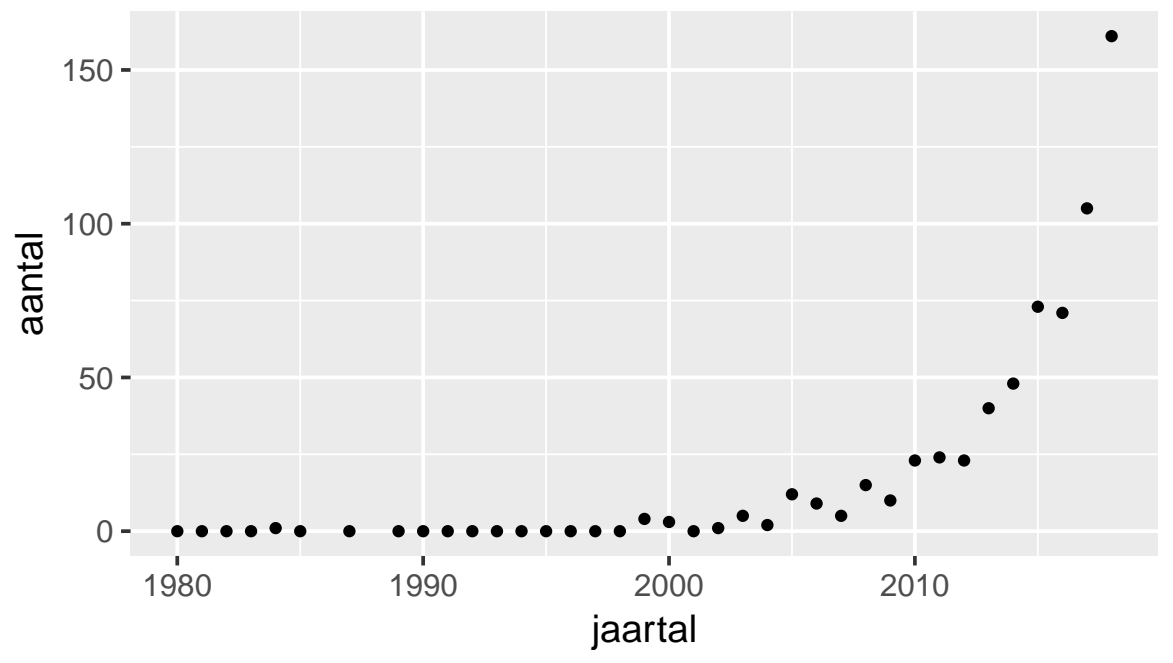

\subsubsection{Trendberekening}

Methode: Poisson

Parameterschattingen

params Estimate Std..Error statistic p.value

1 Intercept $-4.69873790 .35400123-13.27322 \quad 3.310227 \mathrm{e}-40$

2 cJaar $0.2541695 \quad 0.01018113 \quad 24.96477 \quad 1.476123 \mathrm{e}-137$

Trend Zuid-Europese libellensoorten zuidelijke keizerlibel

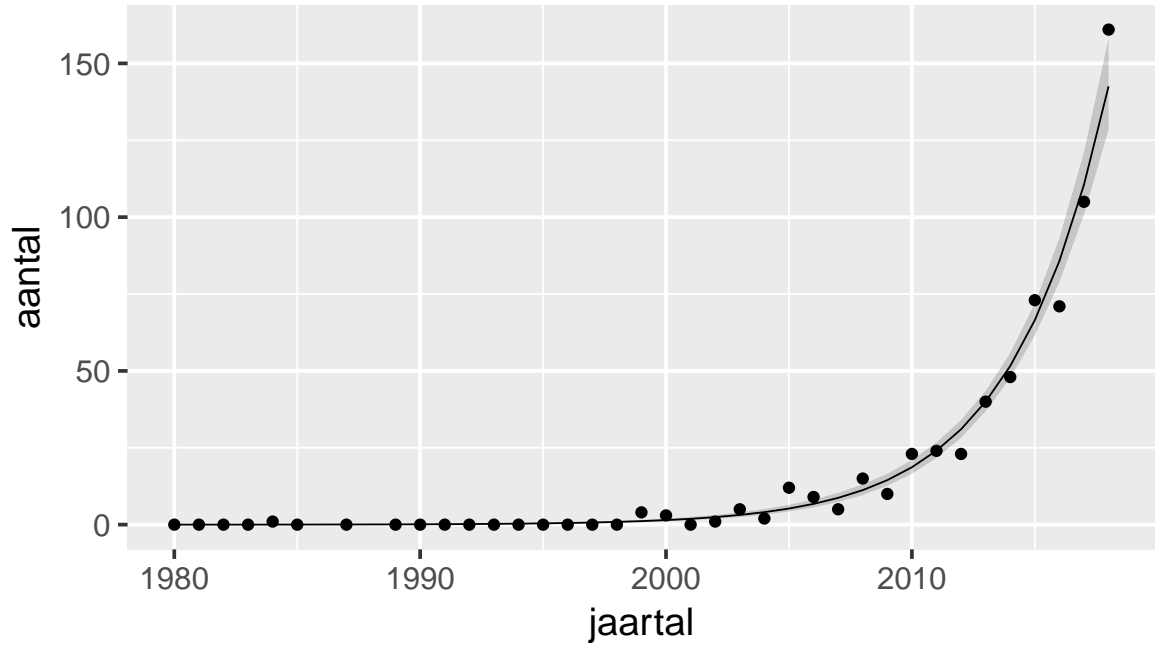

\subsubsection{Verwachting 2020}

$[207,271]$

\subsubsection{Conclusie}

Significante exponentiële toename. 


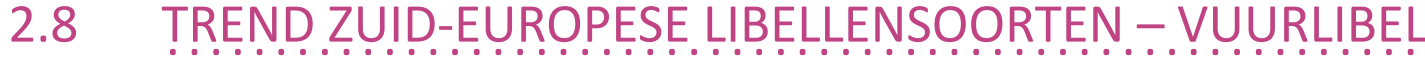

\subsubsection{Ruwe data}

\begin{tabular}{rr}
\hline Jaartal & aantal \\
\hline 1980 & 0.00 \\
1981 & 0.00 \\
1982 & 0.00 \\
1983 & 0.00 \\
1984 & 0.00 \\
1985 & 0.00 \\
1987 & 1.00 \\
1989 & 1.00 \\
1990 & 1.00 \\
1991 & 4.00 \\
1992 & 5.00 \\
1993 & 6.00 \\
1994 & 20.00 \\
1995 & 13.00 \\
1996 & 10.00 \\
1997 & 12.00 \\
1998 & 4.00 \\
1999 & 26.00 \\
2000 & 25.00 \\
2001 & 14.00 \\
2002 & 26.00 \\
2003 & 53.00 \\
2004 & 25.00 \\
2005 & 35.00 \\
2006 & 70.00 \\
2007 & 55.00 \\
2008 & 116.00 \\
2009 & 132.00 \\
2010 & 138.00 \\
2011 & 96.00 \\
2012 & 140.00 \\
2013 & 197.00 \\
2014 & 177.00 \\
2015 & 190.00 \\
2016 & 214.00 \\
2017 & 313.00 \\
2018 & 384.00 \\
\hline \hline &
\end{tabular}




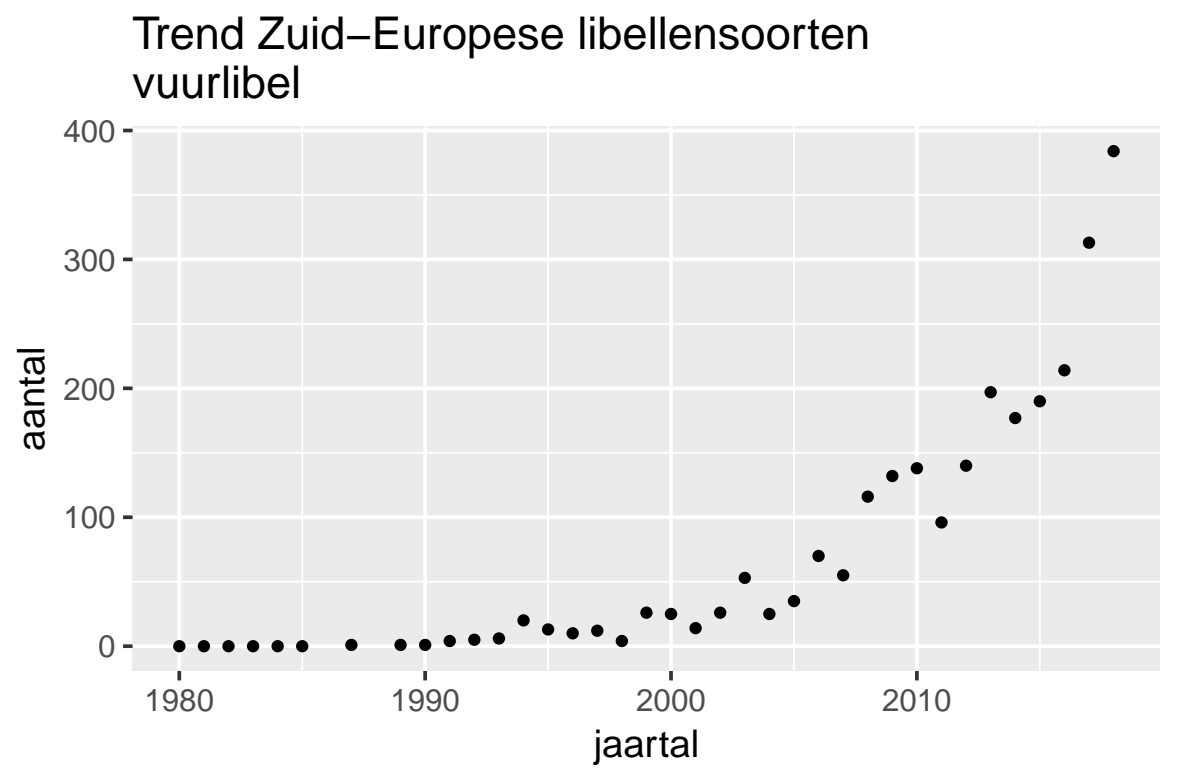

\subsubsection{Trendberekening}

Methode: Poisson smoother

Parameterschattingen

params Estimate Std..Error statistic p.value

1 Intercept $2.724629 \quad 0.103393 \quad 26.35215 \quad 4.849862 \mathrm{e}-153$

$2 \mathrm{~s}$ (Jaartal) $2.735099 \quad 2.940116 \quad 2065.27432 \quad 0.000000 \mathrm{e}+00$

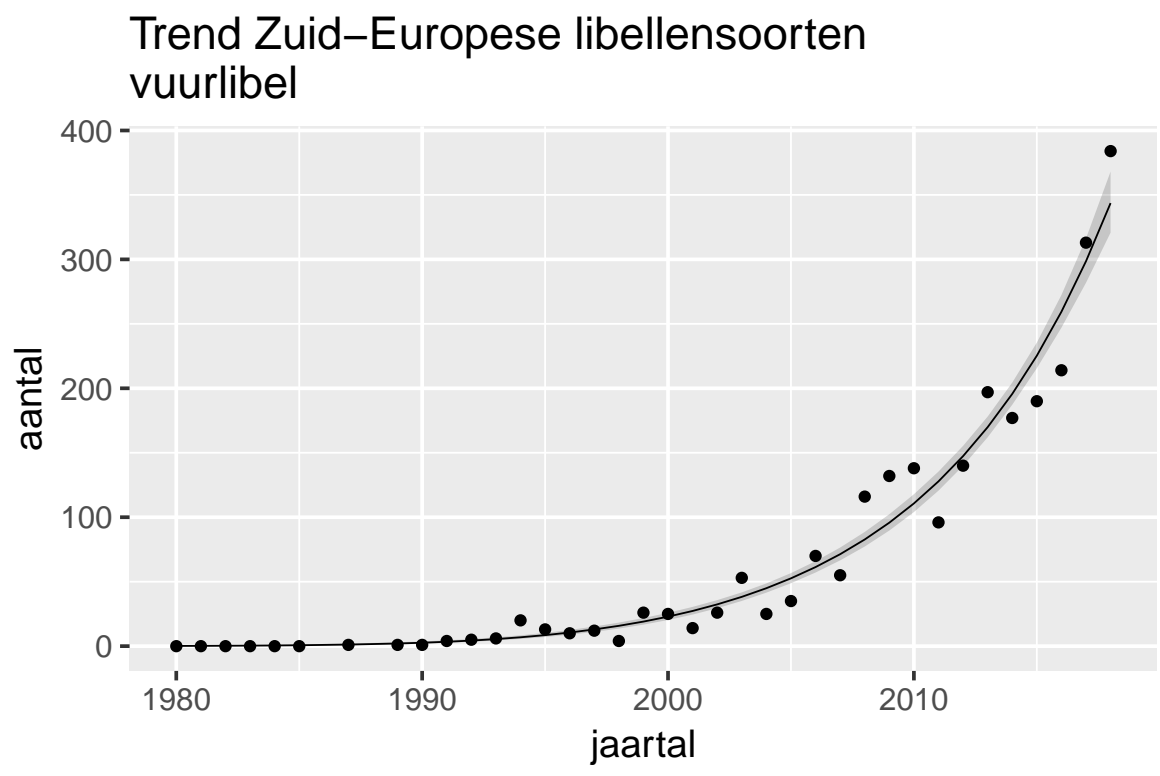

\subsubsection{Verwachting 2020}

$[414,501]$

\subsubsection{Conclusie}

Significante exponentiële toename. 


\subsection{TREND ZUID-EUROPESE LIBELLENSOORTEN - ZUIDELIJKE OEVERLIBEL}

\subsubsection{Ruwe data}

\begin{tabular}{rr}
\hline Jaartal & aantal \\
\hline 1980 & 0.00 \\
1981 & 0.00 \\
1982 & 0.00 \\
1983 & 0.00 \\
1984 & 0.00 \\
1985 & 0.00 \\
1987 & 0.00 \\
1989 & 0.00 \\
1990 & 0.00 \\
1991 & 0.00 \\
1992 & 0.00 \\
1993 & 0.00 \\
1994 & 1.00 \\
1995 & 1.00 \\
1996 & 1.00 \\
1997 & 1.00 \\
1998 & 0.00 \\
1999 & 0.00 \\
2000 & 0.00 \\
2001 & 2.00 \\
2002 & 1.00 \\
2003 & 2.00 \\
2004 & 6.00 \\
2005 & 1.00 \\
2006 & 8.00 \\
2007 & 3.00 \\
2008 & 6.00 \\
2009 & 8.00 \\
2010 & 5.00 \\
2011 & 6.00 \\
2012 & 11.00 \\
2013 & 13.00 \\
2014 & 17.00 \\
2015 & 14.00 \\
2016 & 22.00 \\
\hline & 20.00 \\
2017 & 43.00 \\
\hline
\end{tabular}


Trend Zuid-Europese libellensoorten zuidelijke oeverlibel

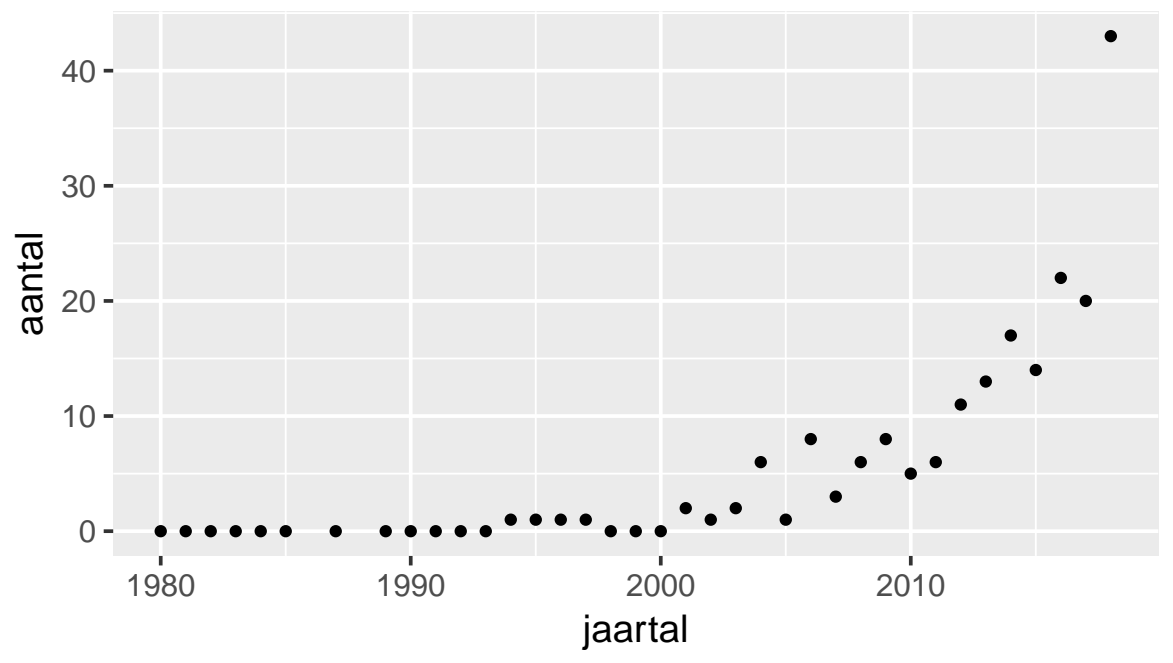

\subsubsection{Trendberekening}

Methode: Poisson

Parameterschattingen

params Estimate Std..Error statistic p.value

1 Intercept $-3.6698755 \quad 0.47245785-7.767625 \quad 7.997122 \mathrm{e}-15$

2 cJaar $0.1886384 \quad 0.01404911 \quad 13.427074 \quad 4.196669 \mathrm{e}-41$

Trend Zuid-Europese libellensoorten zuidelijke oeverlibel

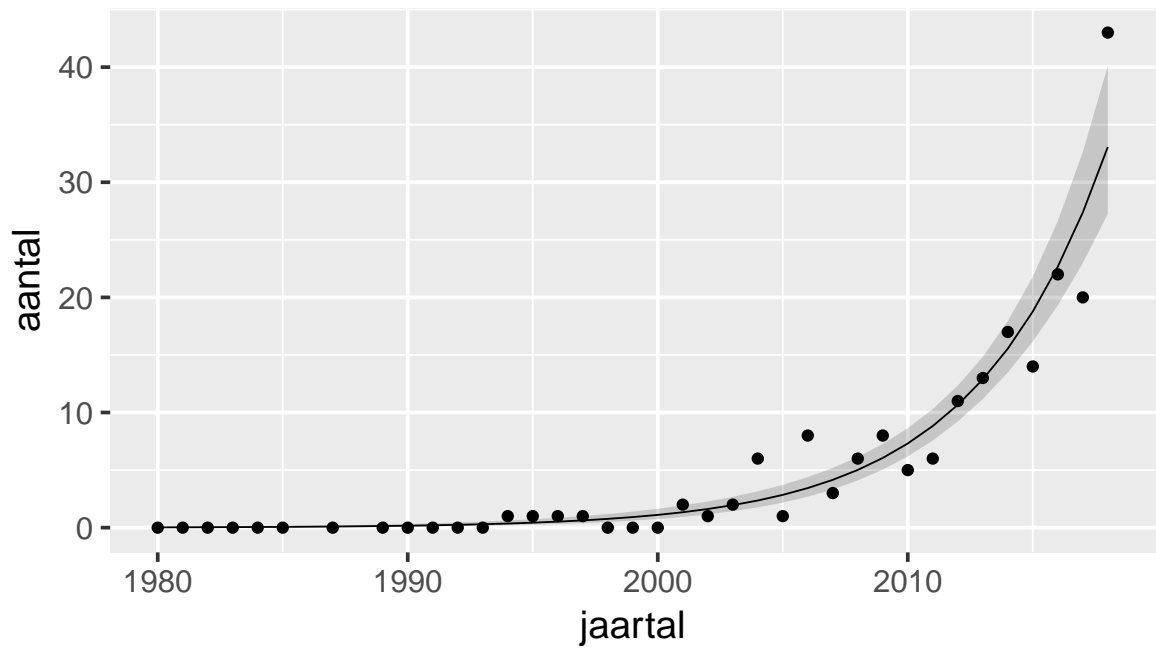

\subsubsection{Verwachting 2020}

$[38.2,60.9]$

\subsubsection{Conclusie}

Significante exponentiële toename. 


\subsection{0}

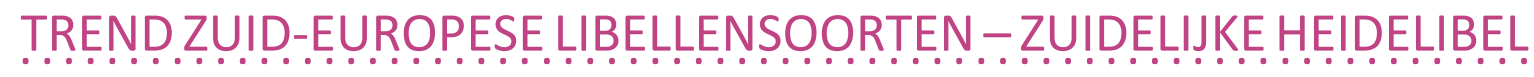

\subsubsection{Ruwe data}

\begin{tabular}{rr}
\hline Jaartal & aantal \\
\hline 1980 & 0.00 \\
1981 & 0.00 \\
1982 & 0.00 \\
1983 & 0.00 \\
1984 & 0.00 \\
1985 & 0.00 \\
1987 & 0.00 \\
1989 & 0.00 \\
1990 & 0.00 \\
1991 & 0.00 \\
1992 & 0.00 \\
1993 & 0.00 \\
1994 & 0.00 \\
1995 & 0.00 \\
1996 & 0.00 \\
1997 & 0.00 \\
1998 & 0.00 \\
1999 & 0.00 \\
2000 & 2.00 \\
2001 & 1.00 \\
2002 & 0.00 \\
2003 & 3.00 \\
2004 & 2.00 \\
2005 & 0.00 \\
2006 & 4.00 \\
2007 & 3.00 \\
2008 & 3.00 \\
2009 & 12.00 \\
2010 & 8.00 \\
2011 & 4.00 \\
2012 & 4.00 \\
2013 & 30.00 \\
2014 & 17.00 \\
2015 & 14.00 \\
2016 & 20.00 \\
2017 & 18.00 \\
2018 & 48.00 \\
\hline \hline & \\
\hline
\end{tabular}


Trend Zuid-Europese libellensoorten zuidelijke heidelibel

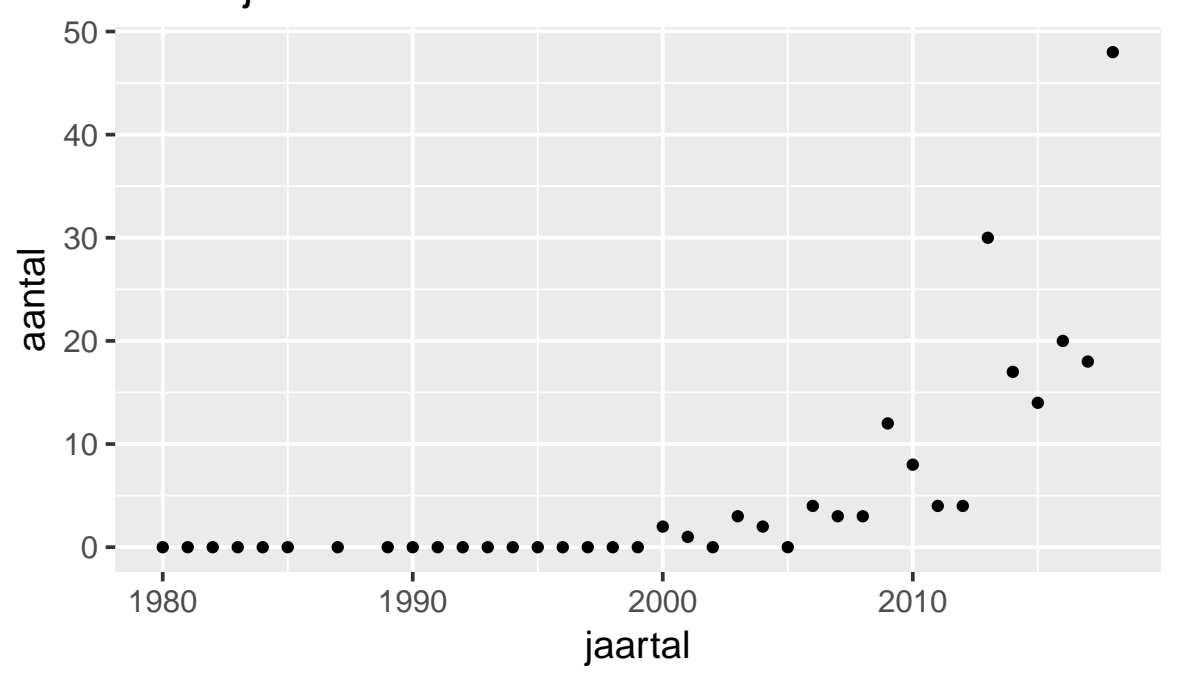

\subsubsection{Trendberekening}

Methode: Poisson smoother

Parameterschattingen

params Estimate Std..Error statistic p.value

1 Intercept $-0.9113901 \quad 0.5266441-1.730562 \quad 8.352996 \mathrm{e}-02$

$2 \mathrm{~s}$ (Jaartal) $1.7114805 \quad 2.0017785155 .529449 \quad 4.523401 \mathrm{e}-34$

Trend Zuid-Europese libellensoorten zuidelijke heidelibel

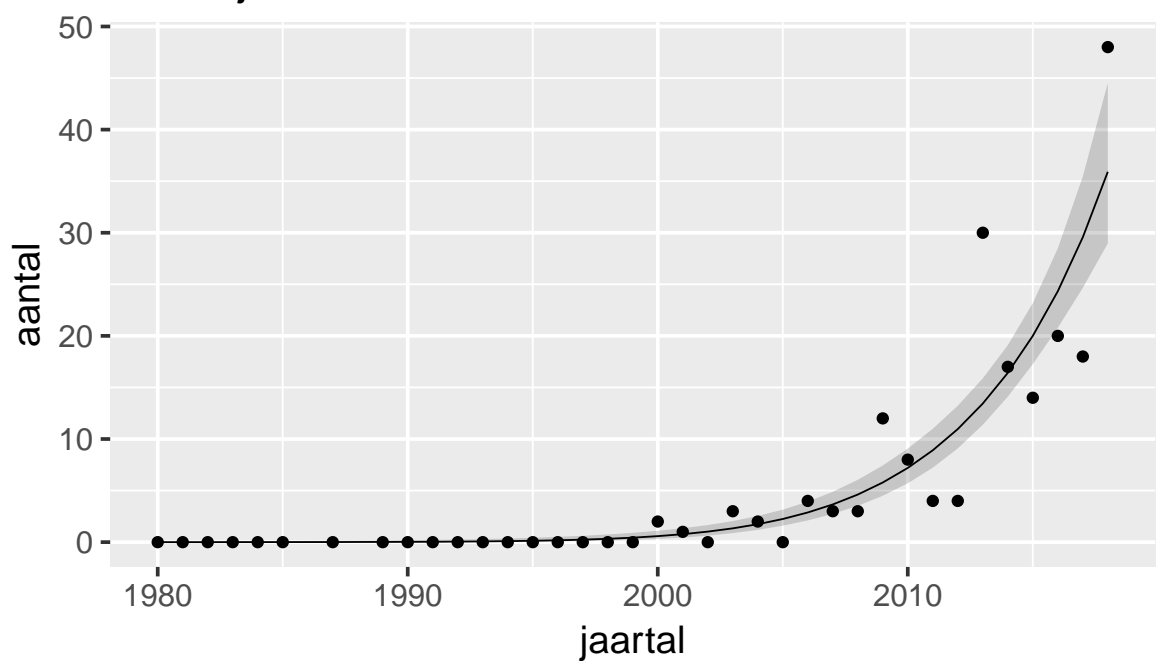

\subsubsection{Verwachting 2020}

$[39.4,71.3]$

\subsubsection{Conclusie}

Significante exponentiële toename. 


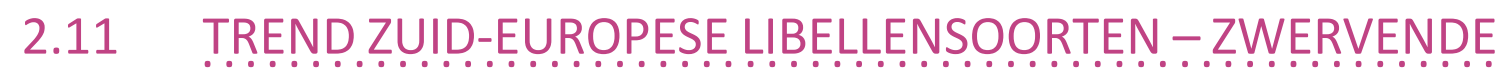
HEIDELIBEL

\subsubsection{Ruwe data}

\begin{tabular}{rr}
\hline Jaartal & aantal \\
\hline 1980 & 0.00 \\
1981 & 0.00 \\
1982 & 0.00 \\
1983 & 0.00 \\
1984 & 1.00 \\
1985 & 0.00 \\
1987 & 0.00 \\
1989 & 1.00 \\
1990 & 0.00 \\
1991 & 1.00 \\
1992 & 2.00 \\
1993 & 2.00 \\
1994 & 2.00 \\
1995 & 1.00 \\
1996 & 16.00 \\
1997 & 8.00 \\
1998 & 5.00 \\
1999 & 10.00 \\
2000 & 8.00 \\
2001 & 0.00 \\
2002 & 16.00 \\
2003 & 19.00 \\
2004 & 8.00 \\
2005 & 9.00 \\
2006 & 36.00 \\
2007 & 56.00 \\
2008 & 22.00 \\
2009 & 75.00 \\
2010 & 20.00 \\
2011 & 22.00 \\
2012 & 106.00 \\
2013 & 82.00 \\
2014 & 43.00 \\
2015 & 150.00 \\
2016 & 44.00 \\
2017 & 220.00 \\
2018 & 55.00 \\
\hline & \\
&
\end{tabular}


Trend Zuid-Europese libellensoorten zwervende heidelibel

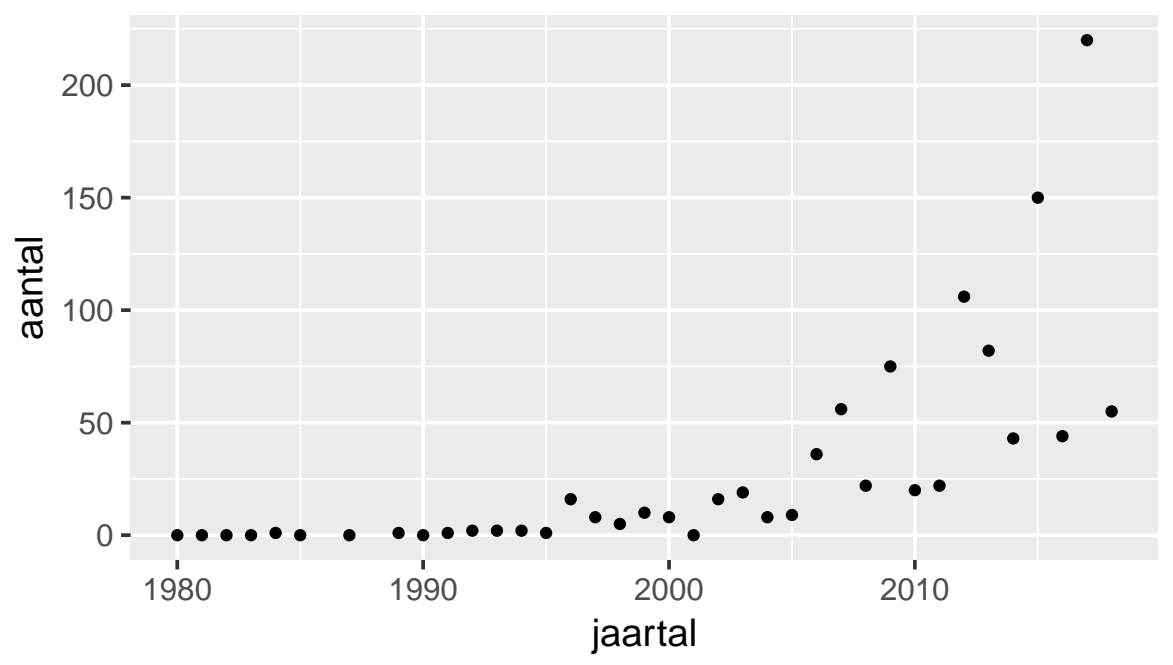

\subsubsection{Trendberekening}

Methode: Poisson smoother

Parameterschattingen

params Estimate Std..Error statistic p.value

1 Intercept $1.953171 \quad 0.1211924 \quad 16.11628 \quad 1.960583 \mathrm{e}-58$

$2 \mathrm{~s}$ (Jaartal) $2.166572 \quad 2.5075346813 .28021 \quad 1.446203 \mathrm{e}-124$

Trend Zuid-Europese libellensoorten

zwervende heidelibel

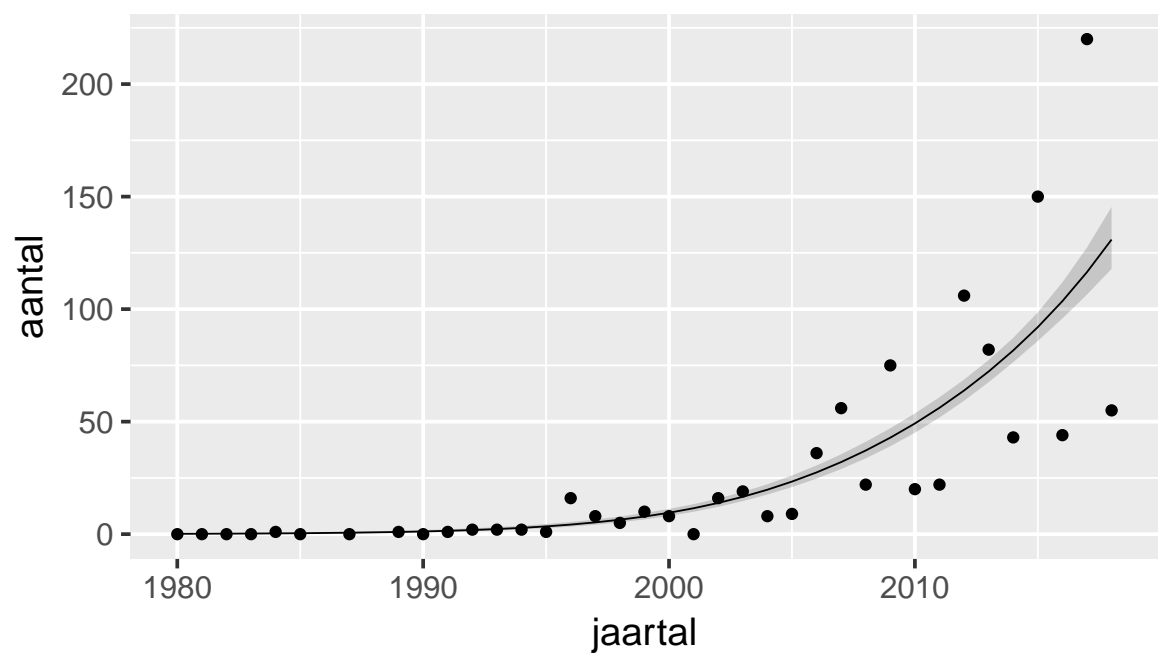

\subsubsection{Verwachting 2020}

$[144,190]$

\subsubsection{Conclusie}

Significante exponentiële toename. 


\subsection{BLADONTWIKKELING - BEUK BLADBEGIN}

\subsubsection{Ruwe data}

\begin{tabular}{rr}
\hline Jaartal & week van het jaar \\
\hline 2002 & 17.30 \\
2003 & 17.70 \\
2004 & 16.80 \\
2005 & 18.30 \\
2006 & 17.50 \\
2007 & 16.80 \\
2008 & 17.80 \\
2009 & 16.50 \\
2010 & 18.30 \\
2011 & 16.20 \\
2012 & 18.00 \\
2013 & 18.10 \\
2014 & 16.00 \\
2015 & 17.40 \\
2016 & 17.40 \\
2017 & 17.70 \\
2018 & 16.20 \\
\hline \hline
\end{tabular}

\section{Bladontwikkeling}

beuk bladbegin

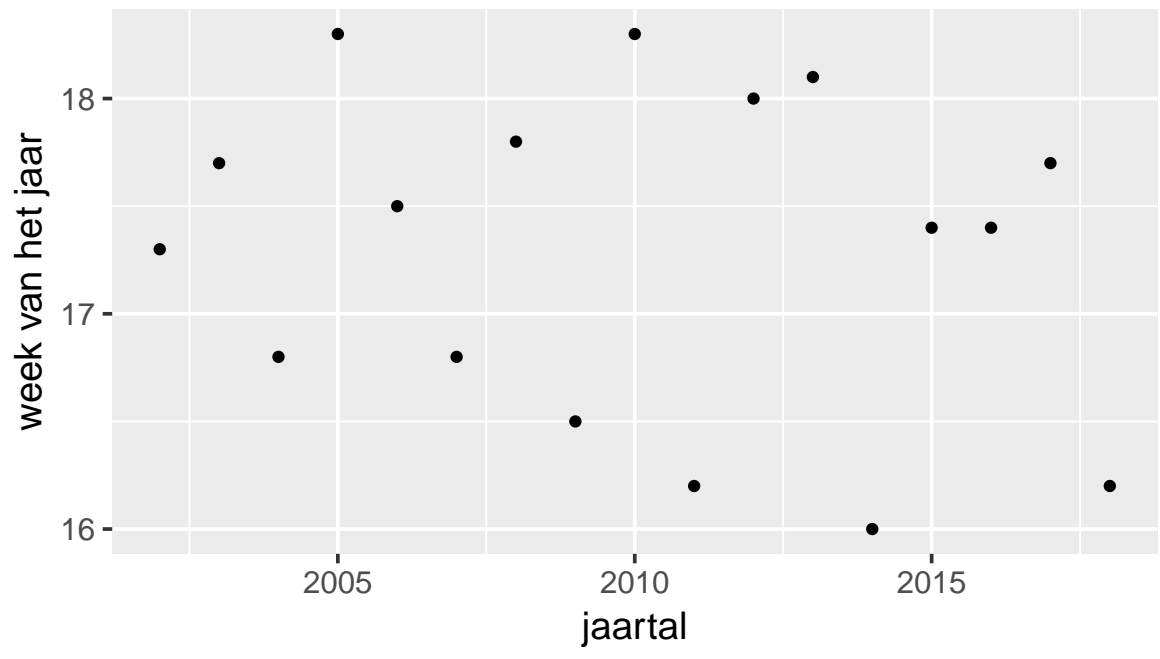

\subsubsection{Trendberekening}

Methode: Lineair

Parameterschattingen

params Estimate Std..Error statistic p.value 1 Intercept $17.52352887 \quad 0.3531609649 .6190996 \quad 4.720543 \mathrm{e}-18$

$2 \quad$ cJaar $-0.02867642 \quad 0.03764708-0.7617168 \quad 4.580389 \mathrm{e}-01$ 


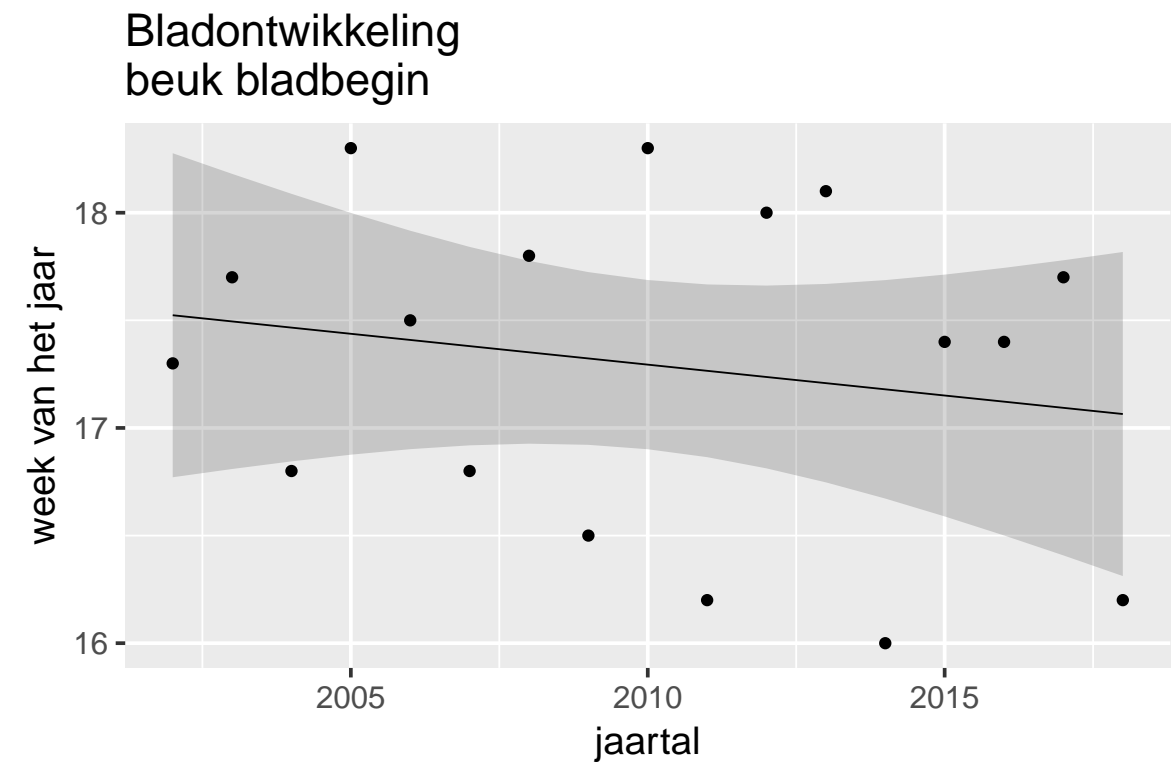

2.12.3 Verwachting 2020

$[15.2,18.9]$

\subsubsection{Conclusie}

Geen significante trend $(p=0.458)$. 


\subsection{BLADONTWIKKELING - BEUK BLADVOL}

\subsubsection{Ruwe data}

\begin{tabular}{rr}
\hline Jaartal & week van het jaar \\
\hline 2002 & 20.00 \\
2003 & 18.90 \\
2004 & 18.30 \\
2005 & 19.60 \\
2006 & 18.90 \\
2007 & 17.80 \\
2008 & 18.60 \\
2009 & 17.60 \\
2010 & 19.50 \\
2011 & 17.60 \\
2012 & 19.30 \\
2013 & 19.10 \\
2014 & 17.60 \\
2015 & 18.80 \\
2016 & 19.70 \\
2017 & 19.30 \\
2018 & 17.40 \\
\hline \hline
\end{tabular}

\section{Bladontwikkeling}

beuk bladvol

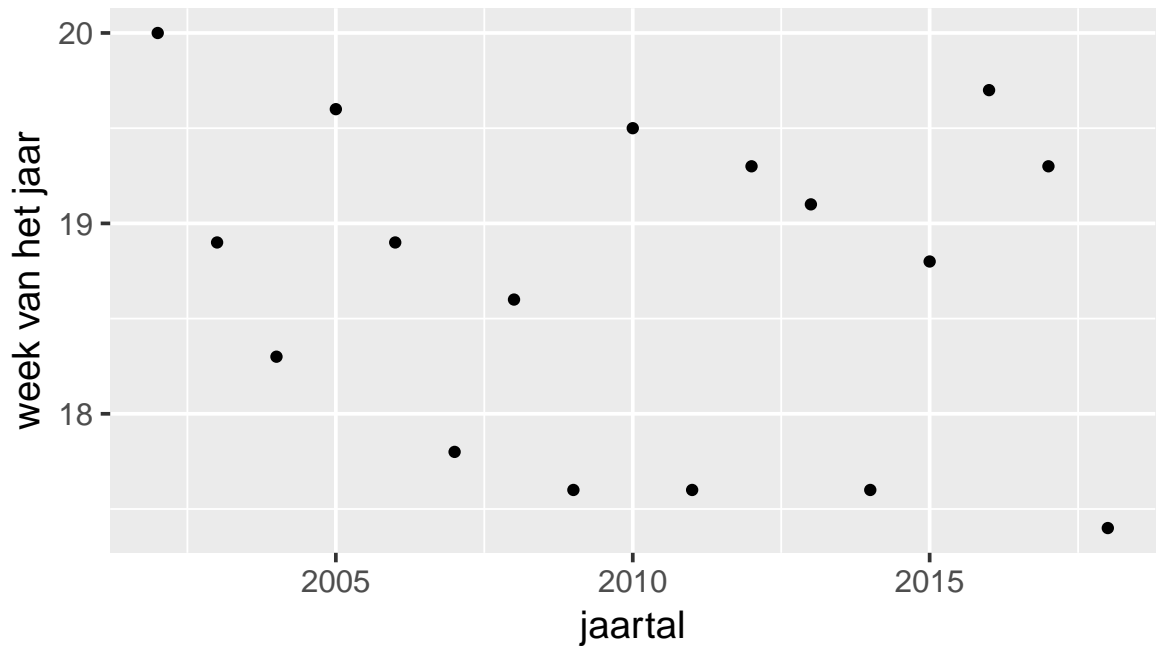

\subsubsection{Trendberekening}

Methode: Lineair

Parameterschattingen

params Estimate Std..Error statistic p.value 1 Intercept $18.970588090 .3970313647 .78108228 .287624 \mathrm{e}-18$

$2 \quad$ cJaar $-0.033088230 .04232369-0.7817899 \quad 4.465035 \mathrm{e}-01$ 
Bladontwikkeling beuk bladvol

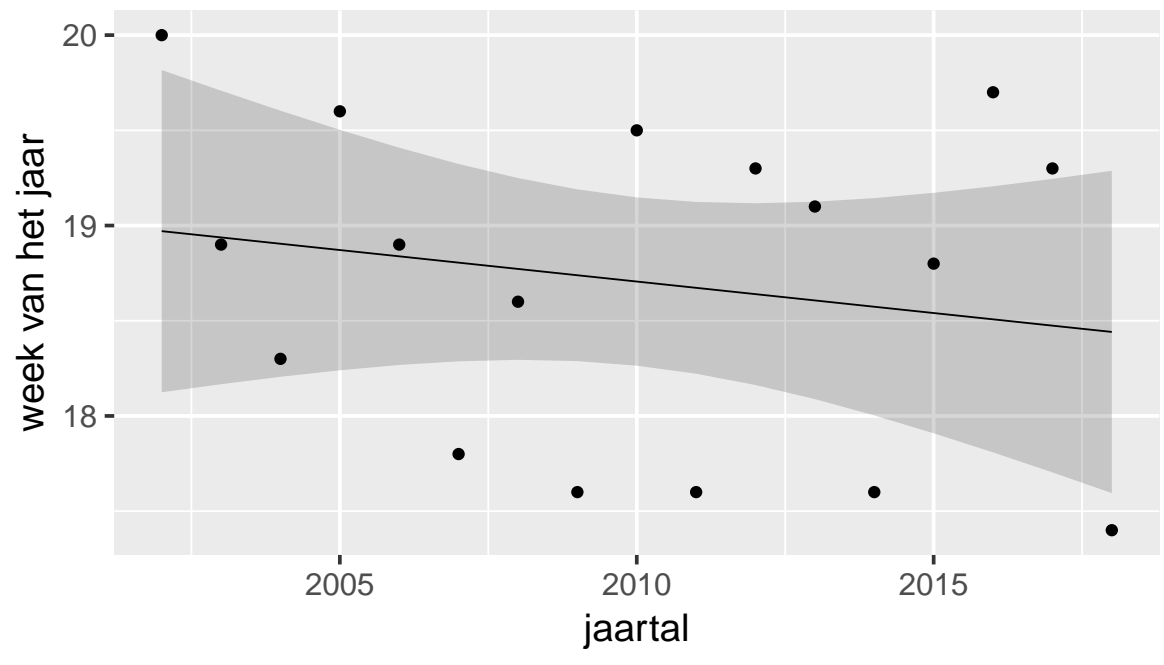

\subsubsection{Verwachting 2020}

$[16.3,20.5]$

\subsubsection{Conclusie}

Geen significante trend $(p=0.342)$. 


\subsection{BLADONTWIKKELING - EIK BLADBEGIN}

\subsubsection{Ruwe data}

\begin{tabular}{rr}
\hline Jaartal & week van het jaar \\
\hline 2003 & 17.50 \\
2004 & 18.10 \\
2005 & 17.80 \\
2006 & 18.50 \\
2007 & 16.20 \\
2008 & 18.00 \\
2009 & 17.10 \\
2010 & 17.40 \\
2011 & 15.70 \\
2013 & 18.80 \\
2014 & 16.30 \\
2015 & 17.60 \\
2016 & 18.20 \\
2017 & 16.90 \\
2018 & 16.30 \\
\hline \hline
\end{tabular}

Bladontwikkeling eik bladbegin

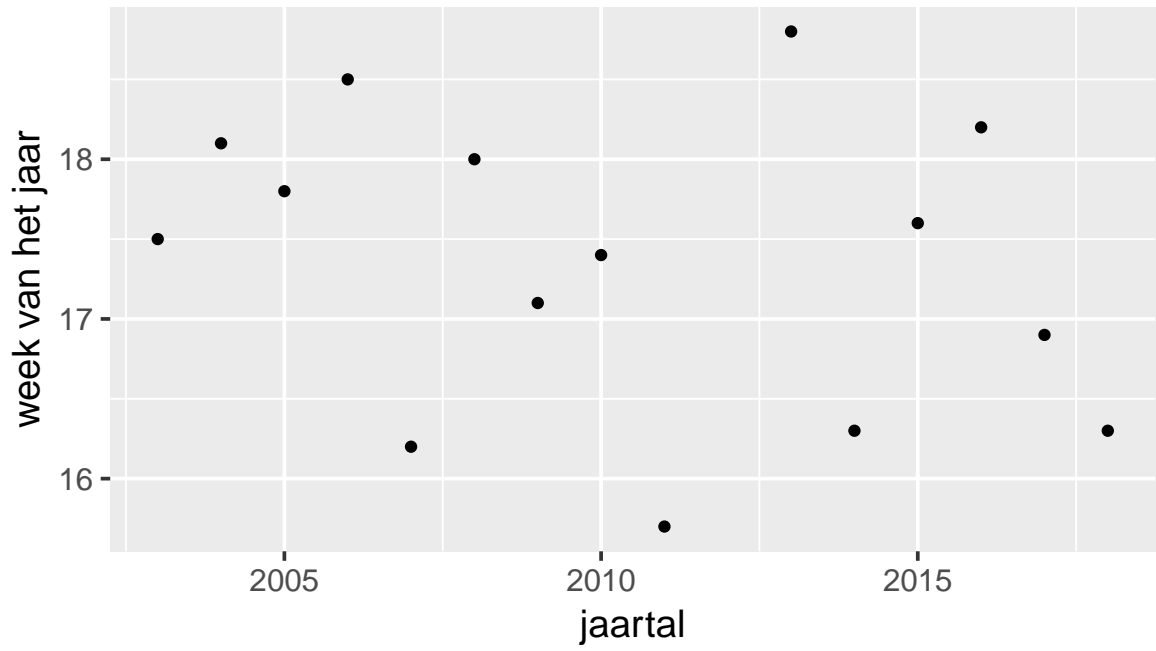

\subsubsection{Trendberekening}

Methode: Lineair

Parameterschattingen

params Estimate Std..Error statistic p.value

1 Intercept $17.72736976 \quad 0.4421423340 .0942604 \quad 5.199556 \mathrm{e}-15$

2 cJaar $-0.04964457 \quad 0.05029977-0.9869742 \quad 3.416697$ e-01 


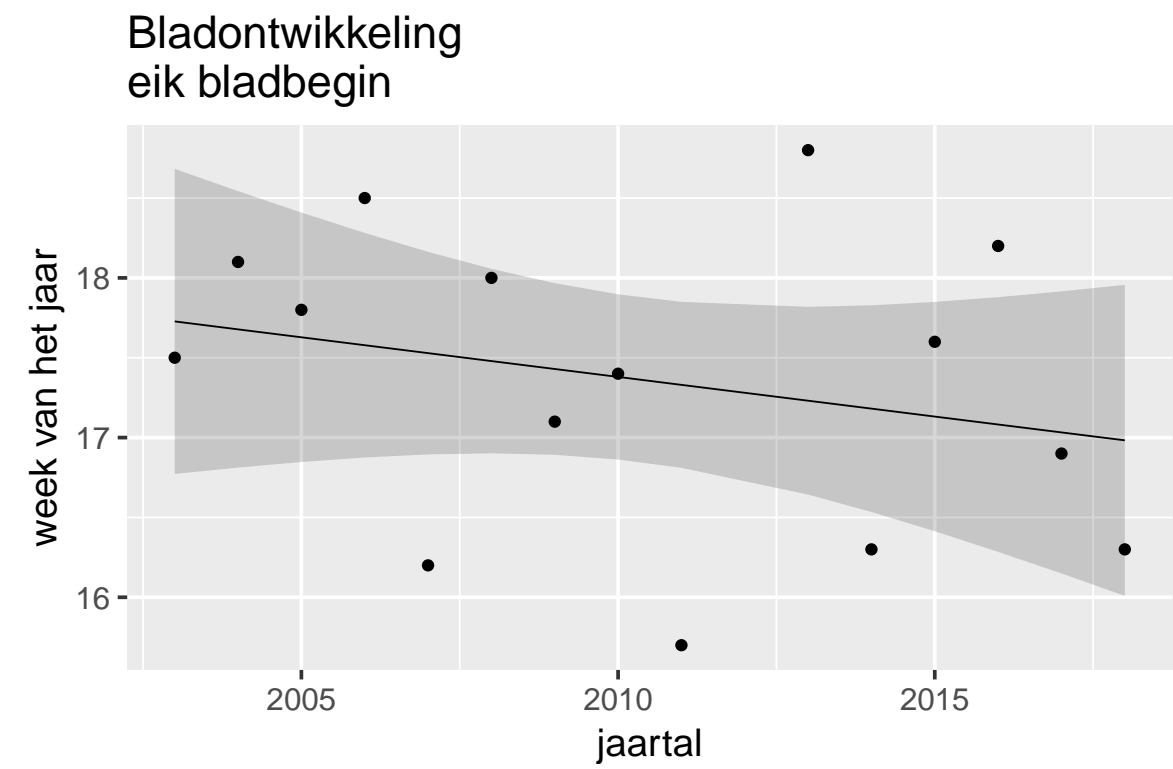

2.14.3 Verwachting 2020

[14.6,19.2]

\subsubsection{Conclusie}

Geen significante trend $(p=0.446)$. 


\subsection{BLADONTWIKKELING - EIK BLADVOL}

\subsubsection{Ruwe data}

\begin{tabular}{rr}
\hline Jaartal & week van het jaar \\
\hline 2003 & 20.20 \\
2004 & 20.40 \\
2005 & 20.80 \\
2006 & 20.00 \\
2007 & 17.80 \\
2008 & 20.10 \\
2009 & 19.90 \\
2010 & 21.00 \\
2013 & 21.40 \\
2014 & 19.00 \\
2014 & 19.00 \\
2015 & 20.00 \\
2016 & 20.20 \\
2017 & 21.00 \\
2018 & 18.30 \\
\hline \hline
\end{tabular}

\section{Bladontwikkeling}

eik bladvol

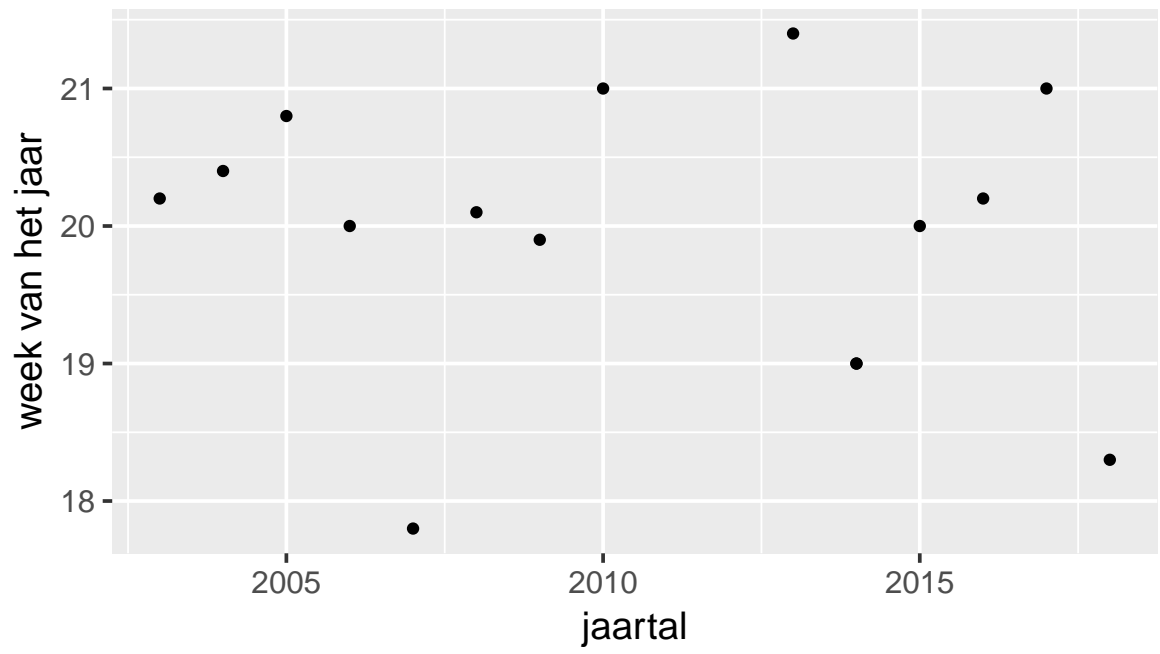

\subsubsection{Trendberekening}

Methode: Lineair

Parameterschattingen

params Estimate Std..Error statistic p.value 1 Intercept $20.15652160 .5042030139 .976996 \quad 5.399814 \mathrm{e}-15$

$2 \quad$ cJaar $-0.02848970 .05599952-0.5087496 .194522 \mathrm{e}-01$ 


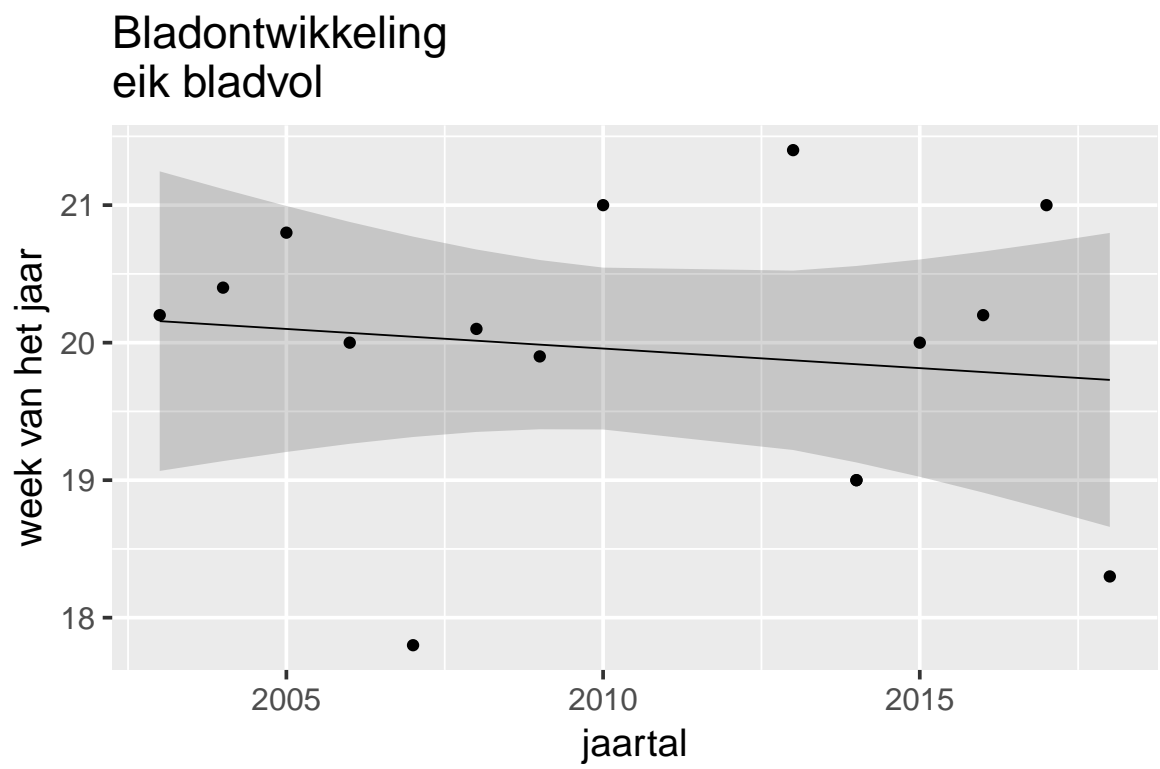

\subsubsection{Verwachting 2020}

$[17.1,22.3]$

\subsubsection{Conclusie}

Geen significante trend $(p=0.619)$. 


\subsection{6}

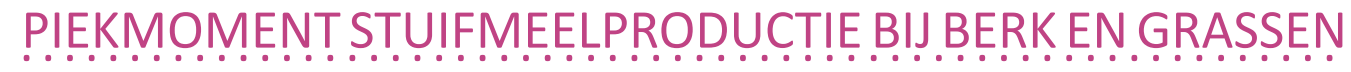
- PIEK BERK

\subsubsection{Ruwe data}

\begin{tabular}{|c|c|}
\hline Jaartal & dag van het jaar \\
\hline 1974 & 95.00 \\
\hline 1975 & 117.00 \\
\hline 1976 & 109.00 \\
\hline 1977 & 122.00 \\
\hline 1978 & 113.00 \\
\hline 1979 & 104.00 \\
\hline 1980 & 122.00 \\
\hline 1981 & 99.00 \\
\hline 1982 & 109.00 \\
\hline 1983 & 114.00 \\
\hline 1984 & 114.00 \\
\hline 1985 & 107.00 \\
\hline 1986 & 122.00 \\
\hline 1987 & 107.00 \\
\hline 1988 & 109.00 \\
\hline 1989 & 99.00 \\
\hline 1990 & 91.00 \\
\hline 1991 & 100.00 \\
\hline 1992 & 102.00 \\
\hline 1993 & 109.00 \\
\hline 1994 & 112.00 \\
\hline 1995 & 112.00 \\
\hline 1996 & 111.00 \\
\hline 1997 & 92.00 \\
\hline 1998 & 112.00 \\
\hline 1999 & 95.00 \\
\hline 2000 & 99.00 \\
\hline 2001 & 121.00 \\
\hline 2002 & 91.00 \\
\hline 2003 & 103.00 \\
\hline 2004 & 104.00 \\
\hline 2005 & 93.00 \\
\hline 2006 & 113.00 \\
\hline 2007 & 102.00 \\
\hline 2008 & 112.00 \\
\hline 2009 & 99.00 \\
\hline 2010 & 102.00 \\
\hline 2011 & 91.00 \\
\hline 2012 & 94.00 \\
\hline 2013 & 113.00 \\
\hline 2014 & 92.00 \\
\hline 2015 & 105.00 \\
\hline 2016 & 101.00 \\
\hline 2017 & 89.00 \\
\hline
\end{tabular}


Piekmoment stuifmeelproductie bij berk en grasser piek berk

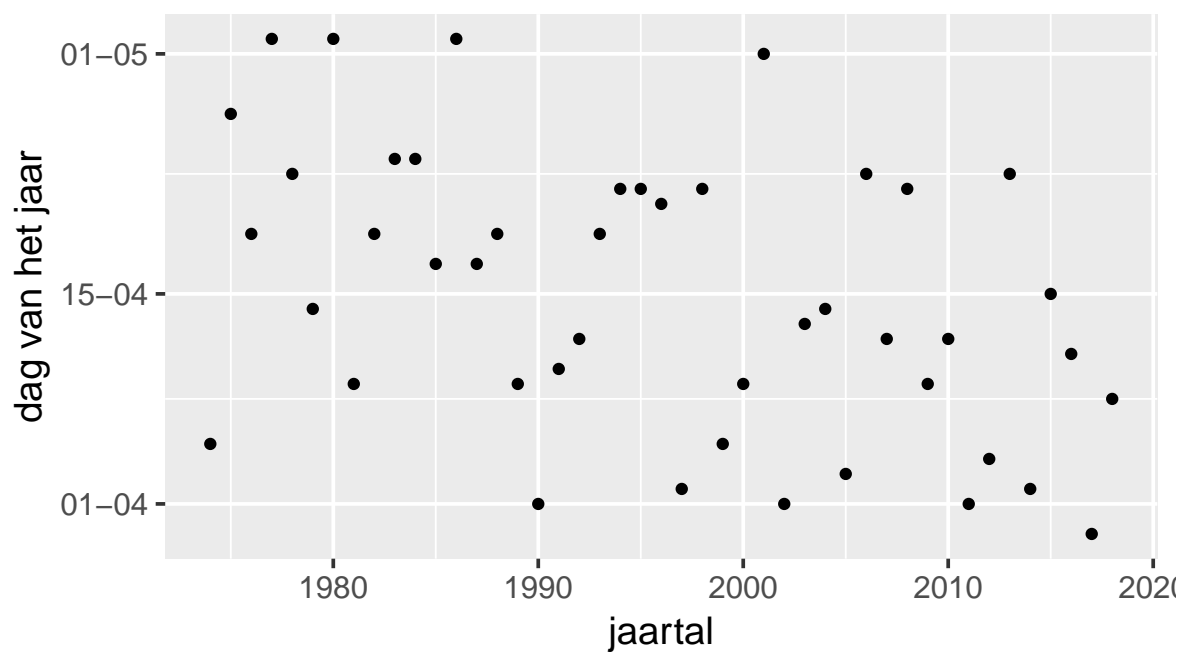

\subsubsection{Trendberekening}

Methode: Lineair

Parameterschattingen

params Estimate Std..Error statistic p.value

1 Intercept $111.74975852 .4899513844 .8802979 .354108 \mathrm{e}-38$

$2 \quad$ cJaar $-0.3118577 \quad 0.09746421 \quad-3.199715 \quad 2.584919 \mathrm{e}-03$

Piekmoment stuifmeelproductie bij berk en grasser piek berk

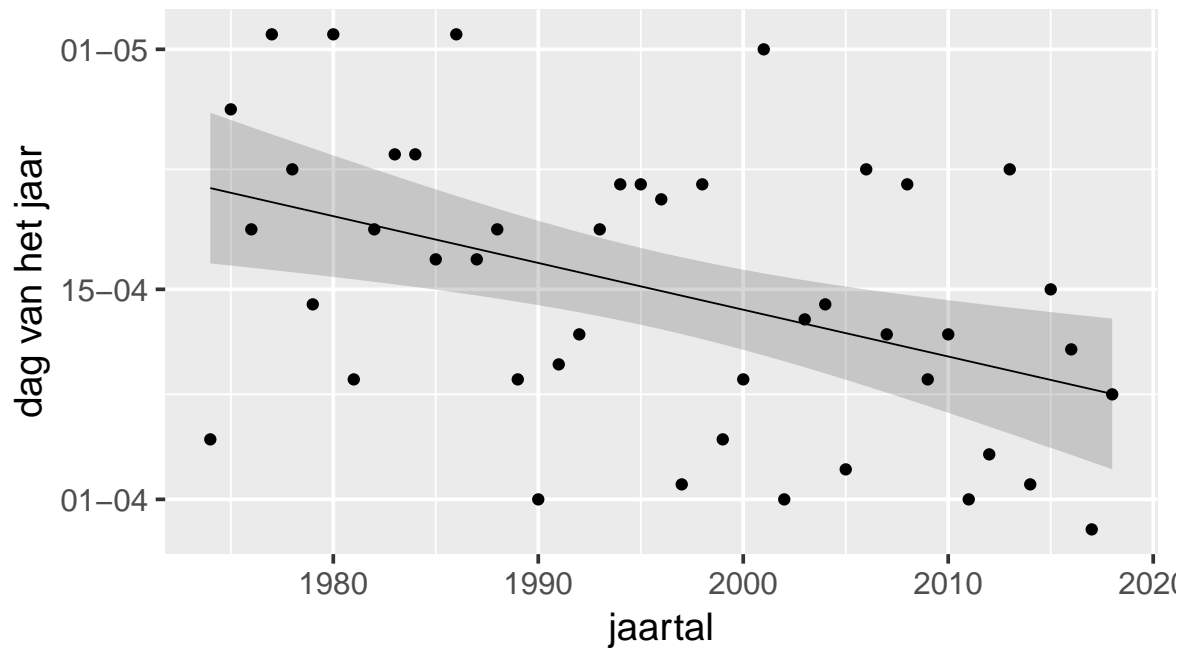

\subsubsection{Verwachting 2020}

$[79.5,115]$

\subsubsection{Conclusie}

Significante afname. 


\subsubsection{Ruwe data}

\begin{tabular}{|c|c|}
\hline Jaartal & dag van het jaar \\
\hline 1974 & 163.00 \\
\hline 1975 & 163.00 \\
\hline 1976 & 160.00 \\
\hline 1977 & 174.00 \\
\hline 1978 & 164.00 \\
\hline 1979 & 160.00 \\
\hline 1980 & 165.00 \\
\hline 1981 & 164.00 \\
\hline 1982 & 156.00 \\
\hline 1983 & 169.00 \\
\hline 1984 & 177.00 \\
\hline 1985 & 153.00 \\
\hline 1986 & 165.00 \\
\hline 1987 & 171.00 \\
\hline 1988 & 165.00 \\
\hline 1989 & 162.00 \\
\hline 1990 & 192.00 \\
\hline 1991 & 186.00 \\
\hline 1992 & 158.00 \\
\hline 1993 & 155.00 \\
\hline 1994 & 173.00 \\
\hline 1995 & 170.00 \\
\hline 1996 & 159.00 \\
\hline 1997 & 160.00 \\
\hline 1998 & 149.00 \\
\hline 1999 & 152.00 \\
\hline 2000 & 160.00 \\
\hline 2001 & 155.00 \\
\hline 2002 & 164.00 \\
\hline 2003 & 157.00 \\
\hline 2004 & 160.00 \\
\hline 2005 & 159.00 \\
\hline 2006 & 162.00 \\
\hline 2007 & 143.00 \\
\hline 2008 & 160.00 \\
\hline 2009 & 149.00 \\
\hline 2010 & 165.00 \\
\hline 2011 & 152.00 \\
\hline 2012 & 150.00 \\
\hline 2013 & 169.00 \\
\hline 2014 & 157.00 \\
\hline 2015 & 174.00 \\
\hline 2016 & 157.00 \\
\hline 2017 & 152.00 \\
\hline
\end{tabular}


Piekmoment stuifmeelproductie bij berk en grasser piek grassen

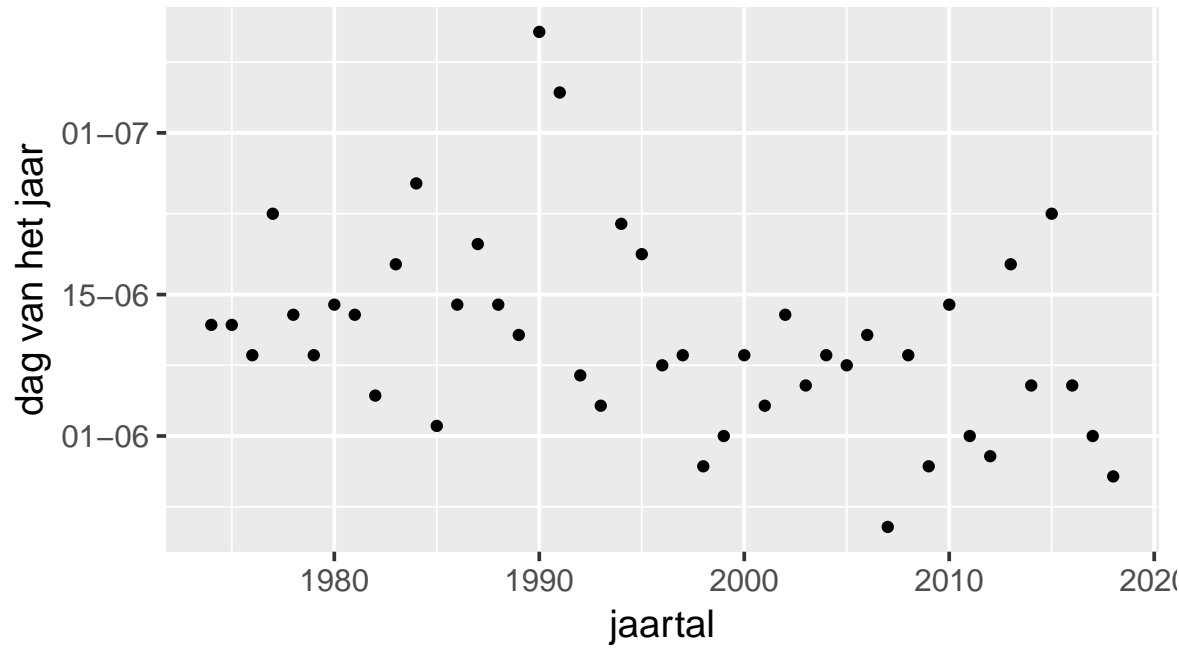

\subsubsection{Trendberekening}

Methode: Lineair

Parameterschattingen

params Estimate Std..Error statistic p.value

1 Intercept $167.6956522 \quad 2.6566621 \quad 63.122688 \quad 4.969081 \mathrm{e}-44$

2 cJaar $-0.2710145 \quad 0.1039898-2.606165 \quad 1.253053 \mathrm{e}-02$

Piekmoment stuifmeelproductie bij berk en grasser piek grassen

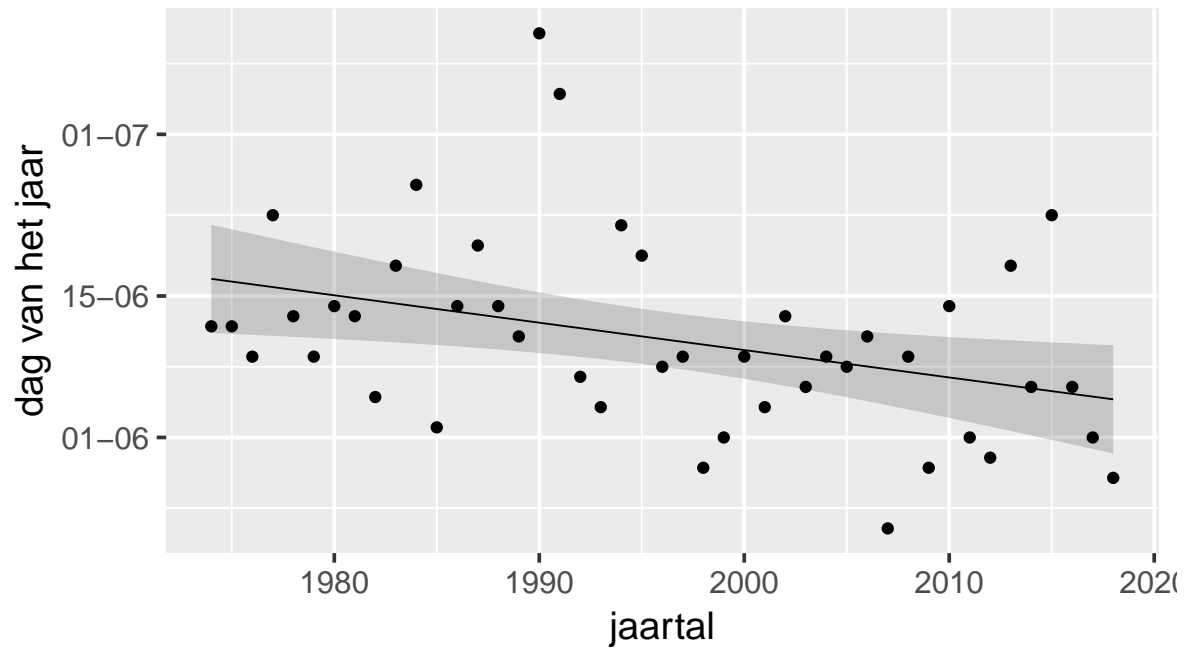

\subsubsection{Verwachting 2020}

$[136,174]$

\subsubsection{Conclusie}

Significante afname. 

UITHEEMSTE PLANTEN

\subsubsection{Ruwe data}

\begin{tabular}{|c|c|c|}
\hline Jaartal & Aandeel & Totaal \\
\hline 1972 & 5.55 & 140 \\
\hline 1973 & 6.43 & 133 \\
\hline 1974 & 6.13 & 137 \\
\hline 1975 & 6.26 & 140 \\
\hline 1976 & 5.79 & 137 \\
\hline 1977 & 5.99 & 137 \\
\hline 1978 & 7.32 & 139 \\
\hline 1979 & 8.06 & 143 \\
\hline 1980 & 8.10 & 141 \\
\hline 1981 & 8.18 & 150 \\
\hline 1982 & 8.69 & 152 \\
\hline 1983 & 9.85 & 146 \\
\hline 1984 & 9.96 & 157 \\
\hline 1985 & 11.36 & 156 \\
\hline 1986 & 11.07 & 155 \\
\hline 1987 & 11.44 & 160 \\
\hline 1988 & 11.45 & 159 \\
\hline 1989 & 10.94 & 151 \\
\hline 1990 & 14.06 & 175 \\
\hline 1991 & 12.29 & 167 \\
\hline 1992 & 11.95 & 164 \\
\hline 1993 & 12.39 & 164 \\
\hline 1994 & 12.82 & 163 \\
\hline 1995 & 11.18 & 159 \\
\hline 1996 & 10.95 & 154 \\
\hline 1997 & 12.52 & 163 \\
\hline 1998 & 13.12 & 159 \\
\hline 1999 & 13.09 & 156 \\
\hline 2000 & 12.22 & 152 \\
\hline 2001 & 12.62 & 154 \\
\hline 2002 & 13.49 & 154 \\
\hline 2003 & 13.73 & 158 \\
\hline 2004 & 13.22 & 164 \\
\hline 2005 & 14.48 & 161 \\
\hline 2006 & 15.26 & 165 \\
\hline 2007 & 15.75 & 160 \\
\hline 2008 & 15.61 & 159 \\
\hline 2009 & 16.37 & 164 \\
\hline 2010 & 14.83 & 157 \\
\hline 2011 & 16.05 & 167 \\
\hline 2012 & 17.41 & 169 \\
\hline 2013 & 17.23 & 175 \\
\hline 2014 & 15.42 & 165 \\
\hline 2015 & 14.40 & 162 \\
\hline
\end{tabular}




\begin{tabular}{lll}
2016 & 14.59 & 152 \\
2017 & 13.94 & 157 \\
2018 & 14.44 & 148 \\
\hline
\end{tabular}

Bedreiging door uitheemse plantensoorten aandeel uitheemse planten

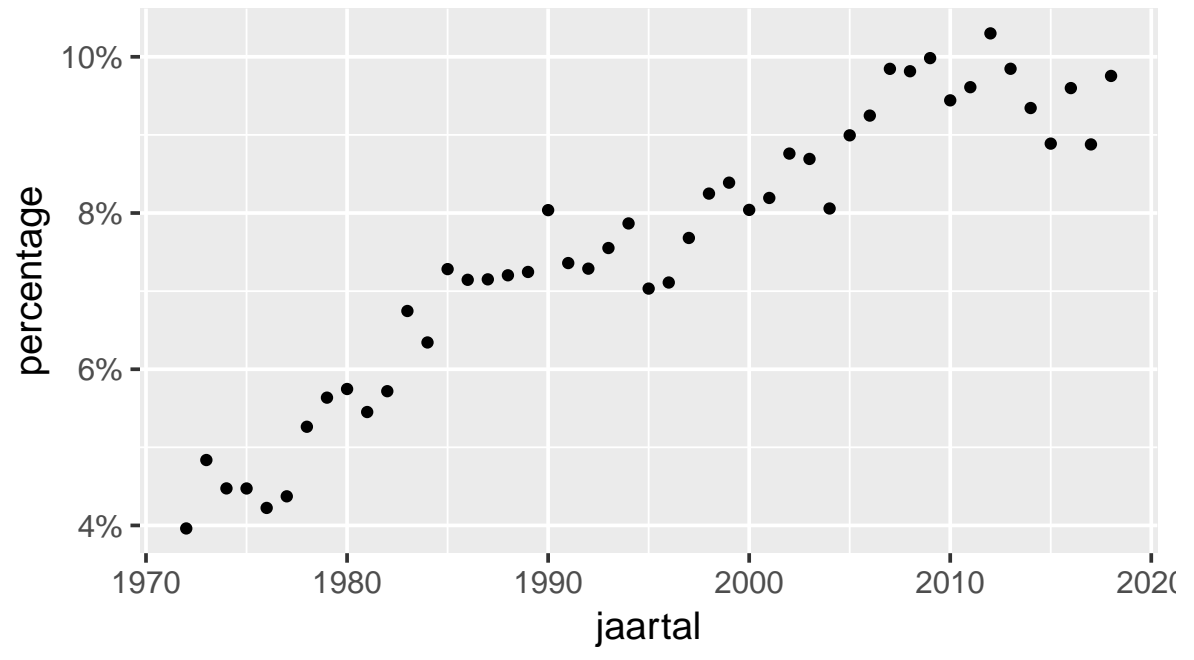

\subsubsection{Trendberekening}

Methode: Binomial

Parameterschattingen

params Estimate Std..Error statistic p.value

1 Intercept $-2.92891303 \quad 0.098540526-29.722929 \quad 3.882278 \mathrm{e}-194$

2 cJaar $0.01755495 \quad 0.003362023 \quad 5.221542 \quad 1.774392 \mathrm{e}-07$

\section{Bedreiging door uitheemse plantensoorten} aandeel uitheemse planten

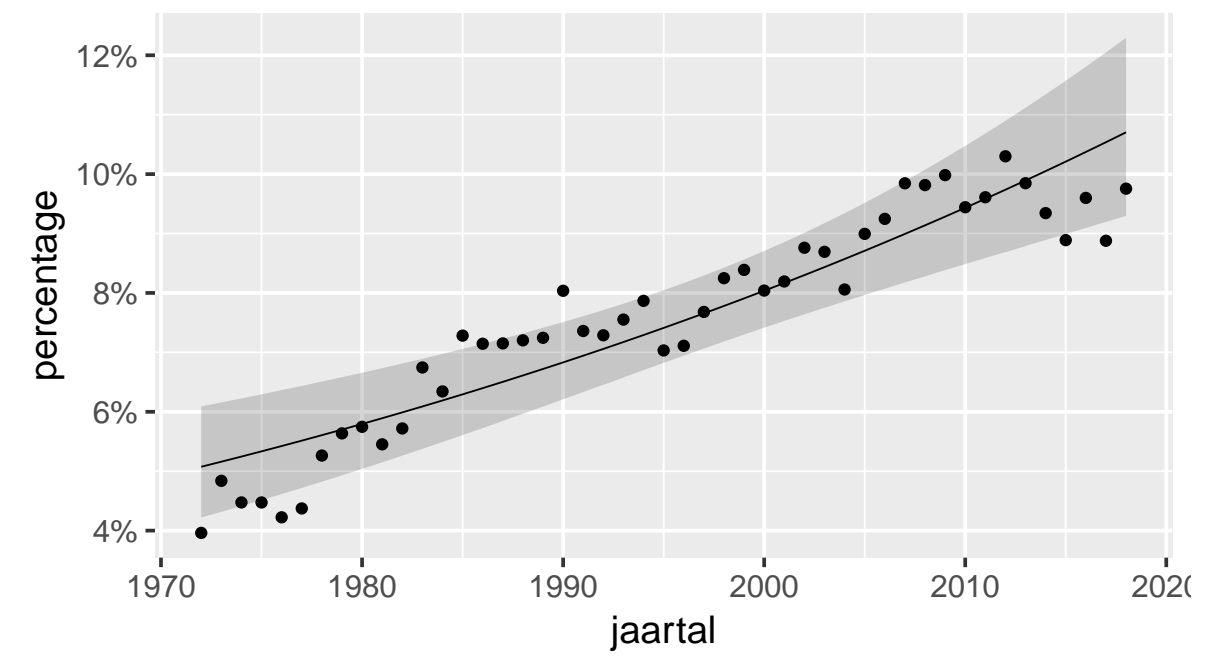

\subsubsection{Verwachting 2020}

$[9.5,12.8]$ 


\subsubsection{Conclusie}

Significante toename. 
